\title{
Complexity growth for topological black holes by holographic method
}

\author{
Koichi Nagasaki \\ Department of Physics, Toho University, \\ Address: 2-2-1 Miyama, Funabashi, Chiba, 274-8510, Japan
}

\begin{abstract}
We consider the growth of the action for black hole spacetime with a fundamental string. Our interest is to find the difference of the behavior between black holes with three different topologies in the scenario of complexity-action conjecture. These black holes have positive, negative and zero curvatures. We would like to calculate the action growth of these systems with a probe fundamental string according to the complexityaction conjecture. We find that for the case where the black holes have the toroidal horizon structure this probe string behaves very differently from the other two cases.
\end{abstract}

\section{Contents}

1 Introduction 1

2 Static topological black holes 3

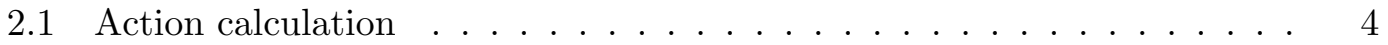

3 Rotating topological black holes 5

3.1 Spherical case . . . . . . . . . . . . . . 7

3.2 Hyperbolic case . . . . . . . . . . . . . . . . . . . . . . 10 10

3.3 Toroidal case . . . . . . . . . . . . . . . . . . 13

4 Discussion

\section{Introduction}

The AdS/CFT correspondence is an important concept in recent theoretical physics [1, 2, 3]. By means of this correspondence, the strong coupling region of gauge theories can be studied by means of gravity method with small coupling method, and vice versa. It will help both for gravitational and gauge theories. Then it is interesting to find a new example of such a correspondence.

One interesting method of this correspondence is the relation between a fundamental string motion in AdS spacetime and the drag force [4, 5]. In these works they added a probe string on the AdS spacetime. An edge of the string is interpreted as a test particle on the dual gauge theory which lives on the infinite boundary of the AdS

\footnotetext{
${ }^{1}$ koichi.nagasaki24@gmail.com
} 
spacetime. This is related to the energy loss of the particle in the quark gluon plasma [6, 7, 8, 9, 10, 11].

A motivation to consider such probe strings is that nonlocal objects are useful to find a new property of the AdS/CFT correspondence, for example in our past work [12], where we confirmed an example of the correspondence between gravity and the gauge sides through a non-local object. Examples of nonlocal objects are Wilson loops, 't Hooft operators, and so on. Especially the string we consider in this paper corresponds to the Wilson loop. By the equation of motion of the string, we will find the embedding of the fundamental string in black hole spacetime and calculate the action. As explained later, there is a conjecture which relates this action to an important quantity to shed the light on the black hole physics.

I would like to explain one more motivation to study non-local operators in black hole spacetime. In theoretical physics, black holes are expected as the fastest scrambler to perform a kind of calculation [13, 14, 15, 16. To quantify such a freedom, recent physics supposes a new quantity. It is "complexity" [17, 18, 19, 20, 21, 22, 23, 24, 25, 26, 27. It has a similar property to entropy as an increasing function of time [28, 29, 30]. Roughly speaking, it counts the number of gates which is needed to cause the quantum development to a given state. In quantum field theory, complexity is studied recently and this definition is revealed in many works $[31,32,33,34,23,35,36$, 37, 38, 39, 40, 41, 42, 27, 43, 44, 45, 46, 47, 48. Geometrical approaches are also studied [49, 50, 51, 52, 53, 54, 55, 56, 57, 58, 59, 60, 61. In this sense, complexity is thought to be a geodesic on the circuit space where gates live. Since holographic complexity is known to have nonlocal properties [62, 63, 62, 64, 65], the nonlocal object will be essential to study the properties of complexity. Complexity of systems with nonlocal operators such as "Wilson loops" are studied also in our past works [66, 67, 68].

Related to the AdS/CFT correspondence, some interesting conjectures for complexity were proposed recently. A reliable candidate is complexity-action (CA) conjecture [69, 70, 71, 72, 73, 74, 75]. Its modified version is reported in [76]. This conjecture asserts the equivalence between complexity and the action calculated in a subspace of the bulk region called "Wheeler DeWitt patch" (WDW). This is the region bounded by null surfaces anchored at the given boundary time. Complexity is expected to be a tool for solving many problems of black holes which includes firewalls, information paradox and so on $[77,78,77$, 80, 81, 82, 83, 84, 85, 86, 87, 88, 89, 90, 91, 92, 93, 94, 95, 96, 97, 98, 99, 100, 101. By this motivation, this conjecture is studied in the various spacetime geometries in many works [102, 85, 86, 103, 104, 105, 106, 107, 108, 109, 110, 111, 112, 113, 114, 115, 116, 117, 118, 119, 120, 121, 122, 123, 124, 125, 126, 127, 128, 129, 130, 131, 132, 133, 134, 135, 136, 137, 138, 139, 140, 141, 142, 143, 144, 145, 146, 147, 148, 149, 150, 151, 152, 153.

We consider in this paper the two sided black holes which exist in AdS spacetime and this spacetime has two conformal field theories on the left and the right side of the AdS boundaries. It is the most studied case for holographic method for black holes. The Penrose diagram of these black holes is depicted in Figure 1. In this figure the horizontal axis represents the radial direction and the vertical axis is the time direction. In this figure the WDW patch anchored at left time $t_{L}$ (the blue region) develops to another patch by time $\delta t$. The difference of these two region comes from the regions $1,2,3$ and 4 in the figure. As time go on, the region 1 and 2 disappear end the region 3 and 4 emerge. As explained in Figure 2 of [70], the contribution from the region 1 is neglected at late times and the contributions from 2 and 3 cancel. Therefore to find the growth of the action on the WDW we only have to integrate the Lagrangian in the region 4 which is bounded by the black hole horizon on the outside and the singularity.

For the transverse geometry of the Penrose diagram, we can take three different types. These are distinguished by the curvature and the metric is given in (3). We call these spacetime with different topologies "topological black holes." The topological 


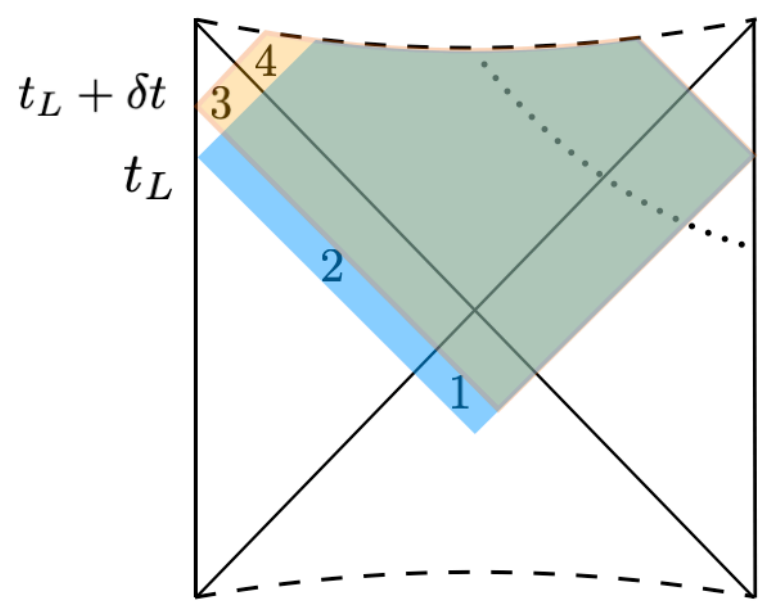

Figure 1: Penrose diagram of the AdS black holes: there are two CFTs on the left and the right sides. The WDW patch shaded in blue at time $t_{L}$ develops to another patch in time $\delta t$. The two diagonal lines represent the black hole horizon. The dashed lines on the upper side and the lower side are singularity. The dotted line on the right side is a fundamental string.

black holes are objects which have interesting applications while hardly studied so far [154, 155, 156, 157, 158, 159]. In this paper we would like to focus on these black holes and find the difference between these topologies.

This paper is organized as follows: In Section 2 we begin with static black hole spacetime with positive, negative and zero curvatures. We solve the equations of motion and find the action growth which is obtained by the integration over the WDW patch. These do not have an interpretation in terms of a drag force because they do not evolve in time. However, we give this calculation for an example for introducing our method for simpler cases. In Section 3 we consider the rotating topological black holes. In Section 4 we conclude this paper by summarizing the results and some discussion.

\section{Static topological black holes}

In this section we consider a fundamental string on the static black hole spacetime. Here the black holes have three different horizon structure whose curvatures are positive, negative or zero. We consider the black hole space time which is asymptotically $\operatorname{AdS}_{d+1}$. The metric is described by [154, 155, 156, 72]

$$
d s_{d+1}^{2}=-f_{k}(r) d t^{2}+\frac{d r^{2}}{f_{k}(r)}+r^{2} d \Sigma_{k, d-1}^{2},
$$

where the metric function $f(r)$ is defined by

$$
f_{k}(r)=k-\frac{2 m}{r^{d-2}}+\frac{r^{2}}{\ell^{2}} \text {. }
$$

In the above parameter $k= \pm 1,0$ distinguishes the topology of the horizon: these are the $(d-1)$-dimensional sphere, hyperboloid and torus. The mass parameter and the AdS radius are denoted by $m$ and $\ell$, respectively. In the following we appropriately rescale the radial coordinate $r$ and put $\ell=1$. The angular part $d \Sigma_{k, d-1}$ is the metric on the $(d-1)$-dimensional Einstein space:

$$
\begin{aligned}
& k=1 \quad \text { sphere }(\mathbb{S}) \quad: \quad d \Sigma_{+1, d-1}^{2}=d \theta^{2}+\sin ^{2} \theta d \Omega_{d-2}^{2}, \quad \theta \in[0, \pi], \\
& k=-1 \quad \text { hyperbolic }(\mathbb{H}) \quad: \quad d \Sigma_{-1, d-1}^{2}=d \theta^{2}+\sinh ^{2} \theta d \Omega_{d-2}^{2}, \quad \theta \in[0, \infty), \\
& k=0 \quad \text { torus }(\mathbb{T}) \quad: \quad d \Sigma_{0, d-1}^{2}=d \theta^{2}+\theta^{2} d \Omega_{d-2}^{2}, \quad \theta \in[0,1] .
\end{aligned}
$$


where for the toroidal case coordinate $\theta$ is periodic.

\subsection{Action calculation}

We consider a static string fixed at $\theta=\theta_{0}$ on the boundary. By changing variables, $y=1 / r$, the metric is

$$
\begin{aligned}
d s_{d+1}^{2} & =\frac{1}{y^{2}}\left(-g_{k}(y) d t^{2}+\frac{d y^{2}}{g_{k}(y)}+d \Sigma_{k, d-1}^{2}\right), \\
g_{k}(y) & =y^{2} f_{k}(1 / y)=1+k y^{2}-2 m y^{d} .
\end{aligned}
$$

In the previous works 67, 68, we considered a string with constant angular velocity around the equator of the sphere. However on the hyperbolic space or torus geometry, there is no such a stationary motion.

Thus we consider here the case where the string is static. This string is not moving then there do not exist an interpretation for the drag force. However in the next section we introduce the angular momentum of the black holes. In this situation the string can occur the drag force. In this section we then treat the static string and black holes as a simple exercise before dealing with the complicated calculation for rotating black holes. The string worldsheet $(\tau, \sigma)$ is embedded as

$$
t=\tau, \quad y=\sigma, \quad \theta=\theta(\sigma) ; \quad \sigma \in[0, \infty) .
$$

The induced metric is

$$
d s_{\text {ind }}^{2}=\frac{1}{\sigma^{2}}\left(-g_{k}(\sigma) d \tau^{2}+\left(\frac{1}{g_{k}(\sigma)}+\theta^{\prime}(\sigma)^{2}\right) d \sigma^{2}\right) .
$$

Note that $d \Omega_{d-2}$ part in (3) vanished since the string does not have the angular momentum and the difference between three topologies appears via only the function $g_{k}$.

The action consists of the gravitational terms for the black hole and the NambuGoto (NG) term for the probe string. The effect of the string to the system is expressed by this NG action

$$
S_{\mathrm{NG}}=T_{\mathrm{str}} \int d \tau d \sigma \mathcal{L}(\sigma),
$$

where

$$
\mathcal{L}(\sigma)=\sqrt{-g_{\text {ind }}(\sigma)}=\frac{1}{\sigma^{2}} \sqrt{1+g_{k}(\sigma) \theta^{\prime}(\sigma)^{2}} .
$$

The equation of motion for $\theta(\sigma)$ is

$$
\frac{d}{d \sigma}\left[\frac{1}{\sigma^{2}} \frac{g_{k}(\sigma) \theta^{\prime}(\sigma)}{\sqrt{1+g_{k}(\sigma) \theta^{\prime}(\sigma)^{2}}}\right]=0 .
$$

It can be integrated easily and gives

$$
\frac{1}{\sigma^{2}} \frac{g_{k}(\sigma) \theta^{\prime}(\sigma)}{\sqrt{1+g_{k}(\sigma) \theta^{\prime}(\sigma)^{2}}}=c
$$

with integration constant $c$. The above equation is solved for $\theta^{\prime}(\sigma)$ as

$$
\theta^{\prime}(\sigma)=\sqrt{\frac{c^{2} \sigma^{4}}{g_{k}(\sigma)\left(g_{k}(\sigma)-c^{2} \sigma^{4}\right)}} .
$$

This denominator of the inside of the square root becomes zero outside of the horizon where $g_{k}-c^{2} \sigma^{4}=0$ while the numerator is non-negative if $c>0$. Then the only possibility that the string penetrates the horizon is $c=0$ and in this case $\theta=$ const. 


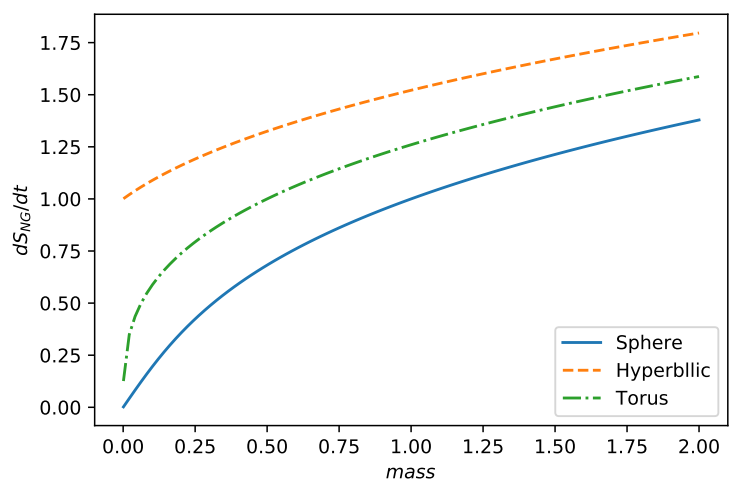

Figure 2: Mass and action growth: $d=3$

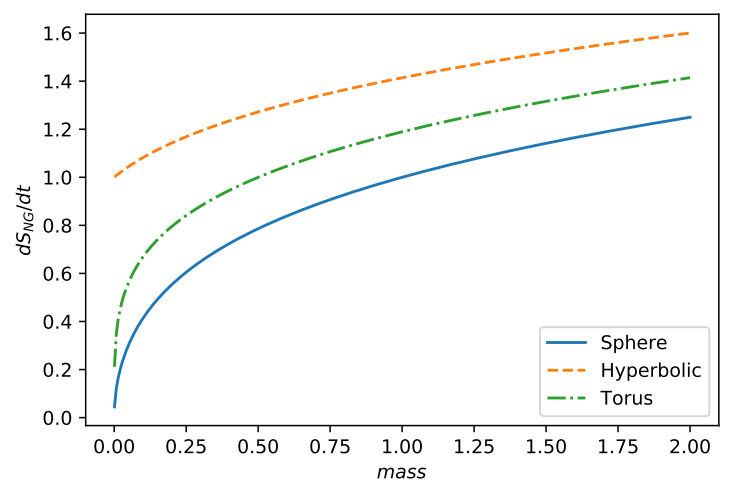

Figure 3: Mass and action growth: $d=4$

WDW action Let us calculate the NG action for Wheeler DeWitt patch in this case. For $\theta^{\prime}=0$ the Lagrangian is simplified as $\mathcal{L}=1 / \sigma^{2}$. To find the growth rate of the action we integrate it inside of the horizon, namely, $y$ is integrated from $y_{\mathrm{h}}:=1 / r_{\mathrm{h}}$ (horizon radius) to the infinity. Then the NG action becomes

$$
\frac{1}{T_{\mathrm{str}}} \frac{d S_{\mathrm{NG}}}{d t}=\int_{y_{\mathrm{h}}}^{\infty} \frac{d \sigma}{\sigma^{2}}=\frac{1}{y_{\mathrm{h}}}=r_{\mathrm{h}} .
$$

It tells the action growth rate is determined by the location of the horizon $r_{\mathrm{h}}$ :

$$
g_{k}\left(y_{\mathrm{h}}\right)=1+k y_{\mathrm{h}}^{2}-2 m y_{\mathrm{h}}^{d}=0 .
$$

The mass dependence is plotted for 3 and 4 dimensions in Figure 2 and Figure 3 . A remarkable point is that there is a non-trivial contribution even in massless case for hyperbolic black holes, as we can see from Equation (14): $0=\left.g_{-1}\left(y_{\mathrm{h}}\right)\right|_{m=0}=1-y_{\mathrm{h}}^{2}$. There exists a non-trivial solution: $y_{\mathrm{h}}=1$.

\section{Rotating topological black holes}

Rotating topological black holes are shown in [160]. The geometries of the $(d+1)$ dimensional rotating black hole with one rotational parameter $a$ are classified into three cases by the curvature $k$. For $k=1$, the spacetime has the spherical horizon:

$$
\begin{aligned}
d s_{\mathrm{KAS}}^{2}=- & \frac{\Delta_{r}}{\rho^{2}}\left(d t-\frac{a}{\Xi} \sin ^{2} \theta d \phi\right)^{2}+\frac{\rho^{2}}{\Delta_{r}} d r^{2}+\frac{\rho^{2}}{\Delta_{\theta}} d \theta^{2} \\
& +\frac{\Delta_{\theta} \sin ^{2} \theta}{\rho^{2}}\left(a d t-\frac{r^{2}+a^{2}}{\Xi} d \phi\right)^{2}+r^{2} \cos ^{2} \theta d \Omega_{1, d-2}^{2}, \\
\Delta_{r}:= & \left(r^{2}+a^{2}\right)\left(r^{2}+1\right)-\frac{2 m}{r^{d-4}}, \quad \Delta_{\theta}:=1-a^{2} \cos ^{2} \theta \\
\rho^{2}:= & r^{2}+a^{2} \cos ^{2} \theta, \quad \Xi:=1-a^{2}
\end{aligned}
$$

where in subscript "KA" denotes Kerr-AdS and "S" means sphere. For the other two cases in the same way we denote them by $d s_{\mathrm{KAH}}^{2}$ and $d s_{\mathrm{KAT}}^{2}$. For $k=-1$, the spacetime 
has the hyperbolic topology:

$$
\begin{aligned}
d s_{\mathrm{KAHI}}^{2}=- & \frac{\Delta_{r}}{\rho^{2}}\left(d t+\frac{a}{\Xi} \sinh ^{2} \theta d \phi\right)^{2}+\frac{\rho^{2}}{\Delta_{r}} d r^{2}+\frac{\rho^{2}}{\Delta_{\theta}} d \theta^{2} \\
& +\frac{\Delta_{\theta} \sinh ^{2} \theta}{\rho^{2}}\left(a d t-\frac{r^{2}+a^{2}}{\Xi} d \phi\right)^{2}+r^{2} \cosh ^{2} \theta d \Omega_{-1, d-2}^{2}, \\
\Delta_{r}:= & \left(r^{2}+a^{2}\right)\left(r^{2}-1\right)-\frac{2 m}{r^{d-4}}, \quad \Delta_{\theta}:=1+a^{2} \cosh ^{2} \theta, \\
\rho^{2}:= & r^{2}+a^{2} \cosh ^{2} \theta, \quad \Xi:=1+a^{2} .
\end{aligned}
$$

For $k=0$, the spacetime has the toroidal topology:

$$
\begin{aligned}
d s_{\mathrm{KAT}}^{2} & =-N^{2} d t^{2}+\frac{\rho^{2}}{\Delta_{r}} d r^{2}+\frac{\rho^{2}}{\Delta_{\theta}} d \theta^{2}+\frac{\Sigma^{2}}{\rho^{2}}(d \phi-\omega d t)^{2}+r^{2} d \Omega_{0, d-2}^{2}, \\
\Delta_{r} & :=a^{2}-\frac{2 m}{r^{d-4}}+r^{4}, \quad \Delta_{\theta}:=1+a^{2} \theta^{4}, \\
\rho^{2} & :=r^{2}+a^{2} \theta^{2}, \\
\Sigma^{2} & :=r^{4} \Delta_{\theta}-a^{2} \theta^{4} \Delta_{r}, \quad \omega:=a \frac{\theta^{2} \Delta_{r}+r^{2} \Delta_{\theta}}{\Sigma^{2}}, \quad N^{2}:=\frac{\rho^{2} \Delta_{\theta} \Delta_{r}}{\Sigma^{2}} .
\end{aligned}
$$

In each case, the parameter $a$ corresponds to the angular momentum per unit mass of the black hole as can been seen in $\omega$ in the torus case. In $a \rightarrow 0$ limit, these surely reproduce the static black holes as follows. The factors of the metric become in this limit,

$$
\begin{aligned}
\text { Sphere }(\mathbb{S}) / \text { hyperbolic }(\mathbb{H}): \Delta_{r} & \rightarrow r^{2}\left(r^{2} \pm 1\right)-\frac{2 m}{r^{d-4}}=r^{2} f(r), \quad \Delta_{\theta} \rightarrow 1, \quad \rho^{2} \rightarrow r^{2}, \\
\Xi & \rightarrow 1, \\
\operatorname{Torus}(\mathbb{T}): \Delta_{r} & \rightarrow r^{4}-\frac{2 m}{r^{d-4}}=r^{2} f(r), \quad \Delta_{\theta} \rightarrow 1, \quad \rho^{2} \rightarrow r^{2}, \\
\Sigma^{2} & \rightarrow r^{4}, \quad \omega \rightarrow 0, \quad N^{2} \rightarrow \frac{r^{4}-2 m / r^{d-4}}{r^{2}}=f(r) .
\end{aligned}
$$

Then the time and radial parts become $-f(r) d t^{2}+d r^{2} / f(r)$ for all three cases and the angular part is

$$
\begin{aligned}
\mathbb{S}: & r^{2} d \theta^{2}+r^{2} \sin ^{2} \theta d \phi^{2}+r^{2} \cos ^{2} \theta d \Omega_{1, d-3}^{2}, \\
\mathbb{H}: & r^{2} d \theta^{2}+r^{2} \sinh ^{2} \theta d \phi^{2}+r^{2} \cosh ^{2} \theta d \Omega_{-1, d-3}^{2}, \\
\mathbb{T}: & r^{2} d \theta^{2}+r^{2} d \phi^{2}+r^{2} d \Omega_{0, d-3}^{2} .
\end{aligned}
$$

These are equal to the metric times radius $r$ shown in (3), respectively. For example, on three-sphere $\sum_{i=0}^{3} x_{i}^{2}=1$, if we choose spherical coordinates

$$
x_{0}=r \cos \psi, x_{1}=r \sin \psi \cos \theta, x_{2}=r \sin \psi \sin \theta \cos \varphi, x_{3}=r \sin \psi \sin \theta \sin \varphi,
$$

the metric is

$$
d s_{S^{3}}^{2}=r^{2}\left(d \psi^{2}+\sin ^{2} \psi d s_{S^{2}}^{2}\right) .
$$

On the other hand, if we choose the Hopf coordinates,

$$
x_{0}=\cos \xi_{1} \sin \eta, x_{1}=\sin \xi_{1} \sin \eta, x_{2}=\cos \xi_{2} \cos \eta, x_{3}=\sin \xi_{2} \cos \eta,
$$

the same metric represented as

$$
d s_{S^{3}}^{2}=d \eta^{2}+\sin ^{2} \eta d \xi_{1}^{2}+\cos ^{2} \eta d \xi_{2}^{2} .
$$


On the hyperbolic case, we can find the similar coordinates by changing $\sin \psi \rightarrow \sinh \psi$ and $\sinh \eta \rightarrow \sinh \eta$ from the sphere case. For the torus case, the metric (3) is the expression by the polar coordinate with radial direction $\theta$ and $(19)$ is the expression by the ordinary flat metric. Then we confirm the equivalence between the previous metric (3) and the present case (19) and thus these Kerr-AdS metric recover the static cases (1) in zero angular momentum limit.

The location of the horizon is depicted in the following figures (Figure 4, Figure 5 and Figure 6). In all cases the horizon radii become small compared with the static black holes.

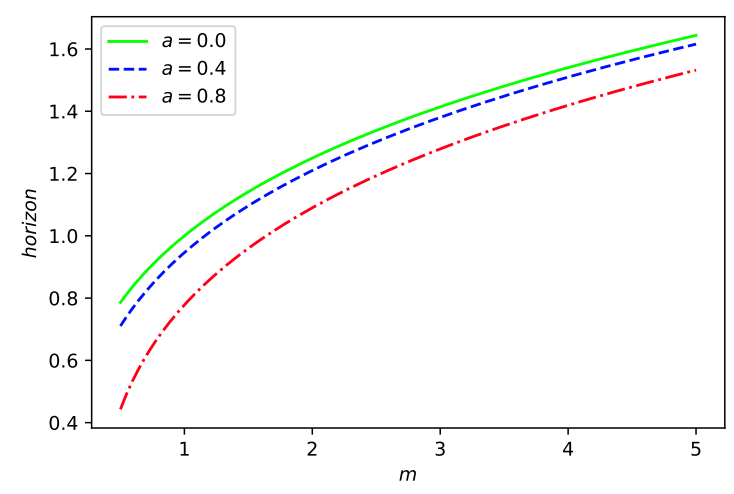

Figure 4: Mass and the horizon $(\mathbb{S}): d=4$

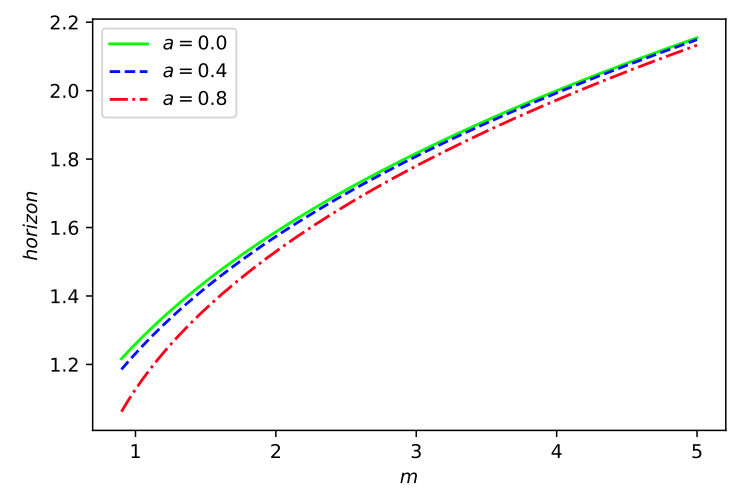

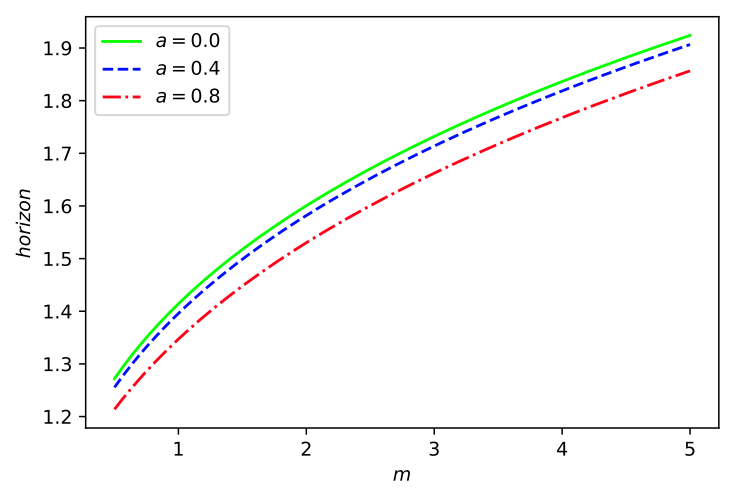

Figure 5: Mass and the horizon $(\mathbb{H}): d=4$

Figure 6: Mass and the horizon $(\mathbb{T}): d=4$

\subsection{Spherical case}

The metric is, if there is no spherical term (we consider a case where the string moves around the equator parametrized by $\phi$ ),

$$
\begin{aligned}
d s_{\mathrm{KAS}}^{2}=- & \frac{\Delta_{r}-\Delta_{\theta} a^{2} \sin ^{2} \theta}{\rho^{2}} d t^{2}+\frac{\rho^{2}}{\Delta_{r}} d r^{2}+\frac{\rho^{2}}{\Delta_{\theta}} d \theta^{2} \\
& +2 \frac{\Delta_{r}-\Delta_{\theta}\left(r^{2}+a^{2}\right)}{\rho^{2} \Xi} a \sin ^{2} \theta d t d \phi+\frac{\Delta_{\theta}\left(r^{2}+a^{2}\right)^{2}-\Delta_{r} a^{2} \sin ^{2} \theta}{\rho^{2} \Xi^{2}} \sin ^{2} \theta d \phi^{2} .
\end{aligned}
$$


Taking the same assumption as before (6), the induced metric on worldsheet $(t=\tau$, $r=r(\sigma), \theta=\theta(\sigma)$ and $\phi=\phi(\sigma))$ is

$$
\begin{aligned}
\left.d s_{\mathrm{KAS}}^{2}\right|_{\text {ind }}= & -\frac{\Delta_{r}-\Delta_{\theta} a^{2} \sin ^{2} \theta}{\rho^{2}} d \tau^{2} \\
& +\left(\frac{\rho^{2}}{\Delta_{r}} r^{\prime 2}+\frac{\rho^{2}}{\Delta_{\theta}} \theta^{\prime 2}+\frac{\Delta_{\theta}\left(r^{2}+a^{2}\right)^{2}-\Delta_{r} a^{2} \sin ^{2} \theta}{\rho^{2} \Xi^{2}} \phi^{\prime 2} \sin ^{2} \theta\right) d \sigma^{2} \\
& +2 \frac{\Delta_{r}-\Delta_{\theta}\left(r^{2}+a^{2}\right)}{\rho^{2} \Xi} a \phi^{\prime} \sin ^{2} \theta d \tau d \sigma .
\end{aligned}
$$

Then the NG action is

$$
\frac{1}{T_{\mathrm{str}}} \frac{d S_{\mathrm{NG}}}{d t}=\int d \sigma \sqrt{\left(\Delta_{r}-\Delta_{\theta} a^{2} \sin ^{2} \theta\right)\left(\frac{r^{\prime 2}}{\Delta_{r}}+\frac{\theta^{\prime 2}}{\Delta_{\theta}}\right)+\frac{\Delta_{r} \Delta_{\theta}}{\Xi^{2}} \phi^{\prime 2} \sin ^{2} \theta}
$$

where as before the integral region is the inside of the horizon. Changing the variables by $y=1 / r$,

$$
\frac{1}{T_{\mathrm{str}}} \frac{d S_{\mathrm{NG}}}{d t}=\int d \sigma \sqrt{\left(\Delta_{r}-\Delta_{\theta} a^{2} \sin ^{2} \theta\right)\left(\frac{y^{\prime 2}}{y^{4} \Delta_{r}}+\frac{\theta^{\prime 2}}{\Delta_{\theta}}\right)+\frac{\Delta_{r} \Delta_{\theta}}{\Xi^{2}} \phi^{\prime 2} \sin ^{2} \theta} .
$$

For notational convenience, we define the following functions.

$$
\begin{aligned}
\Delta_{y} & :=y^{4} \Delta_{r=1 / y}=\left(1+a^{2} y^{2}\right)\left(1+y^{2}\right)-2 m y^{d}, \\
\Theta & :=\Delta_{y}-a^{2} y^{4} \sin ^{2} \theta \Delta_{\theta}, \\
T & :=\frac{\Delta_{y} \Delta_{\theta}}{\Xi^{2}} \sin ^{2} \theta .
\end{aligned}
$$

The action becomes

$$
\frac{1}{T_{\mathrm{str}}} \frac{d S_{\mathrm{NG}}}{d t}=\int d \sigma \mathcal{L}_{\mathbb{S}}, \quad \mathcal{L}_{\mathbb{S}}=\frac{L}{y^{2}}, \quad L=\sqrt{\Theta\left(\frac{y^{\prime 2}}{\Delta_{y}}+\frac{\theta^{\prime 2}}{\Delta_{\theta}}\right)+T \phi^{\prime 2}} .
$$

The equations of motion are

$$
\begin{aligned}
& y^{\prime \prime}-y^{\prime} \frac{d}{d \sigma} \log L+y^{\prime} \frac{d}{d \sigma} \log \left(\frac{\Theta}{y^{2} \Delta_{y}}\right)+\frac{\Delta_{y}}{\Theta}\left(\frac{2 L^{2}}{y}-\frac{1}{2} \frac{\partial L^{2}}{\partial y}\right)=0, \\
& \theta^{\prime \prime}-\theta^{\prime} \frac{d}{d \sigma} \log L+\theta^{\prime} \frac{d}{d \sigma} \log \left(\frac{\Theta}{y^{2} \Delta_{\theta}}\right)-\frac{\Delta_{\theta}}{2 \Theta} \frac{\partial L^{2}}{\partial \theta}=0 \\
& \phi^{\prime \prime}-\phi^{\prime} \frac{d}{d \sigma} \log L+\phi^{\prime} \frac{d}{d \sigma} \log \left(\frac{T}{y^{2}}\right)=0 .
\end{aligned}
$$

We choose the gauge $y^{\prime 2} / \Delta_{y}+\theta^{\prime 2} / \Delta_{\theta}=1$ where $L=\sqrt{\Theta+T \phi^{\prime 2}}$. Then the derivative of $L$ is

$$
\begin{aligned}
L^{\prime} & =\frac{1}{2 L}\left(\Theta^{\prime}+T^{\prime} \phi^{2}+2 T \phi^{\prime} \phi^{\prime \prime}\right)=L A+\frac{T}{L} \phi^{\prime} \phi^{\prime \prime}, \\
A & :=\frac{1}{2 L^{2}}\left(y^{\prime} \partial \Delta_{y}-4 a^{2} y^{3} y^{\prime} \Delta_{\theta} \sin ^{2} \theta-a^{2} y^{4} \theta^{\prime} \partial_{\theta}\left(\Delta_{\theta} \sin ^{2} \theta\right)+T^{\prime} \phi^{2}\right) .
\end{aligned}
$$

In the matrix form the equations are summarized as

$$
\left[\begin{array}{ccc}
1 & & -T y^{\prime} \phi^{\prime} / L^{2} \\
& 1 & -T \theta^{\prime} \phi^{\prime} / L^{2} \\
& & 1-T \phi^{\prime 2} / L^{2}
\end{array}\right]\left[\begin{array}{c}
y^{\prime \prime} \\
\theta^{\prime \prime} \\
\phi^{\prime \prime}
\end{array}\right]=\left[\begin{array}{c}
y^{\prime} A+y^{\prime} B_{y}+C_{y} \\
\theta^{\prime} A+\theta^{\prime} B_{\theta}+C_{\theta} \\
\phi^{\prime} A+\phi^{\prime} B_{\phi}
\end{array}\right]
$$


where we defined

$$
\begin{array}{rlrl}
B_{y} & :=-\frac{d}{d \sigma} \log \left(\frac{\Theta}{y^{2} \Delta_{y}}\right), & B_{\theta} & :=-\frac{d}{d \sigma} \log \left(\frac{\Theta}{y^{2} \Delta_{\theta}}\right), \quad B_{\phi}:=-\frac{d}{d \sigma} \log \left(\frac{T}{y^{2}}\right), \\
C_{y}:=-\frac{\Delta_{y}}{\Theta}\left(\frac{2 L^{2}}{y}-\frac{1}{2} \frac{\partial L^{2}}{\partial y}\right), & C_{\theta}:=\frac{\Delta_{\theta}}{2 \Theta} \frac{\partial L^{2}}{\partial \theta} .
\end{array}
$$

By multiplying the inverse matrix, the equation is solved for the second derivative terms:

$$
\left[\begin{array}{c}
y^{\prime \prime} \\
\theta^{\prime \prime} \\
\phi^{\prime \prime}
\end{array}\right]=\frac{1}{1-T \phi^{\prime 2} / L^{2}}\left[\begin{array}{ccc}
1-T \phi^{\prime 2} / L^{2} & 0 & T y^{\prime} \phi^{\prime} / L^{2} \\
0 & 1-T \phi^{\prime 2} / L^{2} & T \theta^{\prime} \phi^{\prime} / L^{2} \\
0 & 0 & 1
\end{array}\right]\left[\begin{array}{c}
y^{\prime} A+y^{\prime} B_{y}+C_{y} \\
\theta^{\prime} A+\theta^{\prime} B_{\theta}+C_{\theta} \\
\phi^{\prime} A+\phi^{\prime} B_{\phi}
\end{array}\right]
$$

In the above the each factor is

$$
\begin{aligned}
B_{y} & =\frac{2 y^{\prime}}{y}-\frac{\Theta^{\prime}}{\Theta}+\frac{y^{\prime} \partial \Delta_{y}}{\Delta_{y}}, \quad B_{\theta}=\frac{2 y^{\prime}}{y}-\frac{\Theta^{\prime}}{\Theta}+\frac{\theta^{\prime} \partial \Delta_{\theta}}{\Delta_{\theta}}, \quad B_{\phi}=\frac{2 y^{\prime}}{y}-\frac{T^{\prime}}{T}, \\
C_{y} & =-\frac{\Delta_{y}}{\Theta}\left(\frac{2 L^{2}}{y}-\frac{1}{2} \frac{\partial L^{2}}{\partial y}\right), \quad C_{\theta}=\frac{\Delta_{\theta}}{2 \Theta} \frac{\partial L^{2}}{\partial \theta}, \\
\Theta^{\prime} & =y^{\prime} \partial \Delta_{y}-4 a^{2} y^{3} y^{\prime} \sin ^{2} \theta \Delta_{\theta}-a^{2} y^{4} \theta^{\prime} \partial\left(\sin ^{2} \theta \Delta_{\theta}\right), \\
\frac{T^{\prime}}{T} & =\frac{y^{\prime} \partial \Delta_{y}}{\Delta_{y}}+\frac{\theta^{\prime} \partial\left(\sin ^{2} \theta \Delta_{\theta}\right)}{\left(\sin ^{2} \theta \Delta_{\theta}\right)}, \\
\partial \Delta_{y} & =4 a^{2} y^{3}+2\left(a^{2}+1\right) y-2 d m y^{d-1}, \quad \partial \Delta_{\theta}=a^{2} \sin (2 \theta), \\
\partial\left(\sin ^{2} \theta \Delta_{\theta}\right) & =\sin (2 \theta)\left(1-a^{2} \cos (2 \theta)\right) .
\end{aligned}
$$

and

$$
\begin{aligned}
& \frac{\partial L^{2}}{\partial y}=\frac{\partial}{\partial y}\left(\Theta+T \phi^{\prime 2}\right)=\left(1+\frac{\sin ^{2} \theta \Delta_{\theta}}{\Xi^{2}} \phi^{\prime 2}\right) \partial \Delta_{y}-4 a^{2} y^{3} \sin ^{2} \theta \Delta_{\theta}, \\
& \frac{\partial L^{2}}{\partial \theta}=\frac{\partial}{\partial \theta}\left(\Theta+T \phi^{\prime 2}\right)=\left(-a^{2} y^{4}+\frac{\Delta_{y}}{\Xi^{2}} \phi^{\prime 2}\right) \partial\left(\sin ^{2} \theta \Delta_{\theta}\right) .
\end{aligned}
$$

Boundary condition The boundary condition is by the Neumann boundary condition $d \theta / d y=d \phi / d y=0$ and by the gauge condition $y^{\prime 2} / \Delta_{y}+\theta^{\prime 2} / \Delta_{\theta}=1$ at $y=0$,

$$
1=y^{\prime 2}+\frac{\theta^{\prime 2}}{1-a^{2} \cos ^{2} \theta_{0}}, \quad \therefore\left(y, \theta, \phi, y^{\prime}, \theta^{\prime}, \phi^{\prime}\right) \stackrel{y \rightarrow 0}{\rightarrow}\left(0, \theta_{0}, 0,1,0,0\right) .
$$

The results are plotted in 4-dimensional case. Figure 7 represents the mass dependence for fixed angular momentum $a=0.5$. The boundary condition for $\theta$ is $\theta(0)=\pi / 4$. We found the string does not penetrate the horizon in this case. For example, $y_{\mathrm{h}}=0.62$ for mass $m=5$ and $y_{\mathrm{h}}=0.39$ for mass $m=25$.

The angular momentum dependence is plotted in Figure 8 .

WDW action For $\theta_{0}=0$ the action is

$$
\frac{1}{T_{\mathrm{str}}} \frac{d S_{\mathrm{NG}}}{d t}=\int_{y_{\mathrm{h}}}^{\infty} \frac{d y}{y^{2}}=\frac{1}{y_{\mathrm{h}}}=: x
$$

where $y_{\mathrm{h}}$ is determined by

$$
\Delta_{y}\left(y_{\mathrm{h}}\right)=\left(1+a^{2} y_{\mathrm{h}}^{2}\right)\left(1+y_{\mathrm{h}}^{2}\right)-2 m y_{\mathrm{h}}^{d}=0 .
$$

For $d=4$, by solving $x^{4}+\left(1+a^{2}\right) x^{2}-\left(2 m-a^{2}\right)=0,(x>0)$,

$$
\frac{1}{T_{\mathrm{str}}} \frac{d S_{\mathrm{NG}}}{d t}=\left(\frac{-\left(1+a^{2}\right)+\sqrt{\left(1+a^{2}\right)^{2}+4\left(2 m-a^{2}\right)}}{2}\right)^{1 / 2} .
$$

The mass dependence of the action are plotted in Figure 9 . 

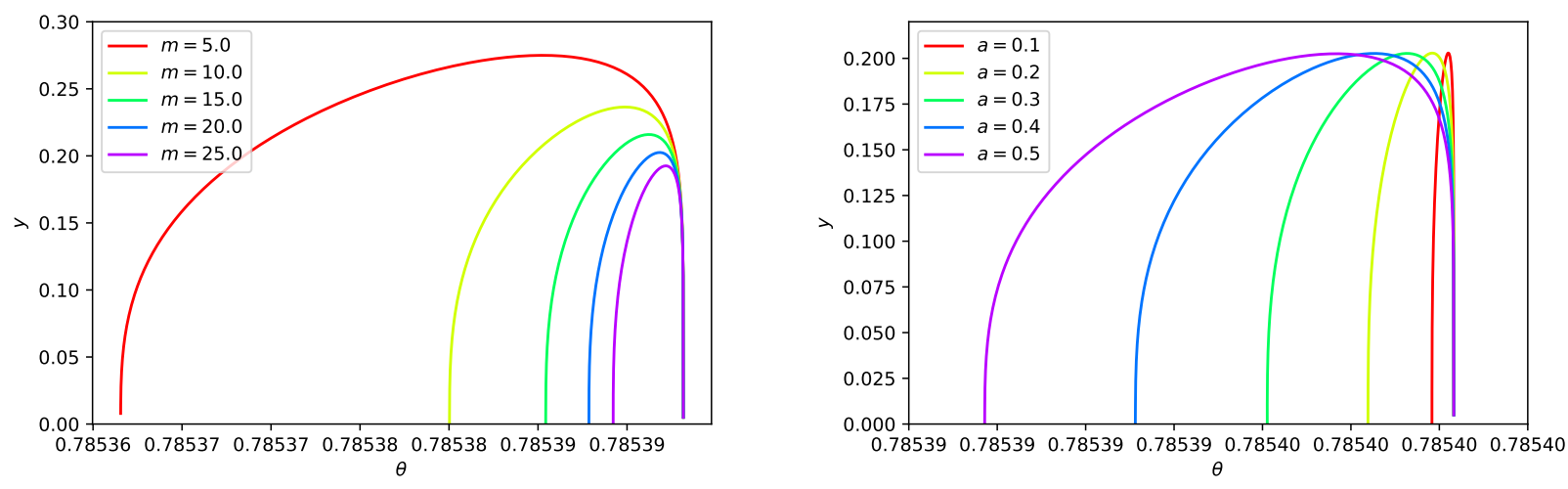

Figure 7: Mass dependence of the string em- Figure 8: Angular momentum dependence of bedding $\left(\mathbb{S}, \theta_{0}=\pi / 4\right)$

the string embedding $(\mathbb{S})$

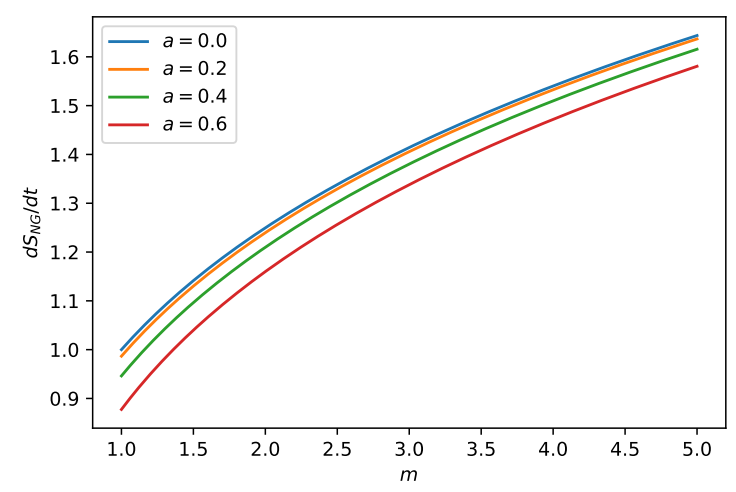

Figure 9: Mass and action growth for various angular momentums $(\mathbb{S})$

\subsection{Hyperbolic case}

The metric is, if there is not spherical term,

$$
\begin{aligned}
d s_{\mathrm{KAH}}^{2}=-\frac{\Delta_{r}-\Delta_{\theta} a^{2} \sinh ^{2} \theta}{\rho^{2}} d t^{2}+ & \frac{\rho^{2}}{\Delta_{r}} d r^{2}+\frac{\rho^{2}}{\Delta_{\theta}} d \theta^{2}-2 \frac{\Delta_{r}+\Delta_{\theta}\left(r^{2}+a^{2}\right)}{\rho^{2} \Xi} a \sinh ^{2} \theta d t d \phi \\
& +\frac{\Delta_{\theta}\left(r^{2}+a^{2}\right)^{2}-\Delta_{r} a^{2} \sinh ^{2} \theta}{\rho^{2} \Xi^{2}} \sinh ^{2} \theta d \phi^{2}
\end{aligned}
$$

On the worldsheet $(t=\tau, r=r(\sigma), \theta=\theta(\sigma)$ and $\phi=\phi(\sigma))$, the induced metric is

$$
\begin{aligned}
d s_{\mathrm{KAH} H}^{2} \mid \text { ind }= & -\frac{\Delta_{r}-\Delta_{\theta} a^{2} \sinh ^{2} \theta}{\rho^{2}} d \tau^{2} \\
& +\left(\frac{\rho^{2}}{\Delta_{r}} r^{\prime 2}+\frac{\rho^{2}}{\Delta_{\theta}} \theta^{\prime 2}+\frac{\Delta_{\theta}\left(r^{2}+a^{2}\right)^{2}-\Delta_{r} a^{2} \sinh ^{2} \theta}{\rho^{2} \Xi^{2}} \sinh ^{2} \theta \phi^{\prime 2}\right) d \sigma^{2} \\
& -2 \frac{\Delta_{r}+\Delta_{\theta}\left(r^{2}+a^{2}\right)}{\rho^{2} \Xi} a \phi^{\prime} \sinh ^{2} \theta d \tau d \sigma .
\end{aligned}
$$

The Lagrangian is, by changing variables $y=1 / r$,

$$
\begin{aligned}
\frac{1}{T_{\mathrm{str}}} \frac{d S_{\mathrm{NG}}}{d t} & =\int d \sigma \sqrt{\left(\Delta_{r}-\Delta_{\theta} a^{2} \sinh ^{2} \theta\right)\left(\frac{r^{\prime 2}}{\Delta_{r}}+\frac{\theta^{\prime 2}}{\Delta_{\theta}}\right)+\frac{\Delta_{r} \Delta_{\theta}}{\Xi^{2}} \phi^{\prime 2} \sinh ^{2} \theta} \\
& =\int d \sigma \sqrt{\left(\Delta_{r}-\Delta_{\theta} a^{2} \sinh ^{2} \theta\right)\left(\frac{1}{y^{4}} \frac{y^{\prime 2}}{\Delta_{r}}+\frac{\theta^{\prime 2}}{\Delta_{\theta}}\right)+\frac{\Delta_{r} \Delta_{\theta}}{\Xi^{2}} \phi^{\prime 2} \sinh ^{2} \theta}
\end{aligned}
$$


For later convenience, we define

$$
\begin{aligned}
\frac{1}{T_{\mathrm{str}}} \frac{d S_{\mathrm{NG}}}{d t} & =\int d \sigma \mathcal{L}_{\mathbb{H}}, \quad \mathcal{L}_{\mathbb{H}}=\frac{L}{y^{2}}, \\
L & :=\sqrt{\left(\Delta_{y}-\Delta_{\theta} a^{2} y^{4} \sinh ^{2} \theta\right)\left(\frac{y^{\prime 2}}{\Delta_{y}}+\frac{\theta^{\prime 2}}{\Delta_{\theta}}\right)+\frac{\Delta_{y} \Delta_{\theta}}{\Xi^{2}} \phi^{\prime 2} \sinh ^{2} \theta} .
\end{aligned}
$$

In the same way as the spherical case, we define

$$
\begin{aligned}
& L=\sqrt{\Theta\left(\frac{y^{\prime 2}}{\Delta_{y}}+\frac{\theta^{\prime 2}}{\Delta_{\theta}}\right)+T \phi^{\prime 2}}, \\
& \Theta:=\Delta_{y}-a^{2} y^{4} \sinh ^{2} \theta \Delta_{\theta}, \quad T:=\frac{\Delta_{y} \Delta_{\theta}}{\Xi^{2}} \sinh ^{2} \theta, \\
& \Delta_{y}:=\left(1+a^{2} y^{2}\right)\left(1-y^{2}\right)-2 m y^{d}, \quad \Delta_{\theta}:=1+a \cosh ^{2} \theta .
\end{aligned}
$$

The equations of motion are

$$
\begin{aligned}
& y^{\prime \prime}-y^{\prime} \frac{d}{d \sigma} \log L+y^{\prime} \frac{d}{d \sigma} \log \left(\frac{\Theta}{y^{2} \Delta_{y}}\right)+\frac{\Delta_{y}}{\Theta}\left(\frac{2 L^{2}}{y}-\frac{1}{2} \frac{\partial L^{2}}{\partial y}\right)=0, \\
& \theta^{\prime \prime}-\theta^{\prime} \frac{d}{d \sigma} \log L+\theta^{\prime} \frac{d}{d \sigma} \log \left(\frac{\Theta}{y^{2} \Delta_{\theta}}\right)-\frac{\Delta_{\theta}}{2 \Theta} \frac{\partial L^{2}}{\partial \theta}=0 \\
& \phi^{\prime \prime}-\phi^{\prime} \frac{d}{d \sigma} \log L+\phi^{\prime} \frac{d}{d \sigma} \log \left(\frac{T}{y^{2}}\right)=0 .
\end{aligned}
$$

We choose the gauge $y^{\prime 2} / \Delta_{y}+\theta^{\prime 2} / \Delta_{\theta}=1, L=\sqrt{\Theta+T \phi^{\prime 2}}$. Then the derivative of $L$ is

$$
\begin{aligned}
L^{\prime} & =L A+\frac{T}{L} \phi^{\prime} \phi^{\prime \prime}, \\
A & :=\frac{1}{2 L^{2}}\left(y^{\prime} \partial \Delta_{y}-4 a^{2} y^{3} y^{\prime} \Delta_{\theta} \sinh ^{2} \theta-a^{2} y^{4} \theta^{\prime} \partial_{\theta}\left(\Delta_{\theta} \sinh ^{2} \theta\right)+T^{\prime} \phi^{2}\right),
\end{aligned}
$$

where we defined

$$
\begin{array}{rlrl}
B_{y} & :=-\frac{d}{d \sigma} \log \left(\frac{\Theta}{y^{2} \Delta_{y}}\right), & B_{\theta} & :=-\frac{d}{d \sigma} \log \left(\frac{\Theta}{y^{2} \Delta_{\theta}}\right), \quad B_{\phi}:=-\frac{d}{d \sigma} \log \left(\frac{T}{y^{2}}\right), \\
C_{y}:=-\frac{\Delta_{y}}{\Theta}\left(\frac{2 L^{2}}{y}-\frac{1}{2} \frac{\partial L^{2}}{\partial y}\right), & C_{\theta}:=\frac{\Delta_{\theta}}{2 \Theta} \frac{\partial L^{2}}{\partial \theta} .
\end{array}
$$

These are explicitly

$$
B_{y}=\frac{2 y^{\prime}}{y}-\frac{\Theta^{\prime}}{\Theta}+\frac{y^{\prime} \partial \Delta_{y}}{\Delta_{y}}, \quad B_{\theta}=\frac{2 y^{\prime}}{y}-\frac{\Theta^{\prime}}{\Theta}+\frac{\theta^{\prime} \partial \Delta_{\theta}}{\Delta_{\theta}}, \quad B_{\phi}=\frac{2 y^{\prime}}{y}-\frac{T^{\prime}}{T} .
$$

In the above

$$
\begin{aligned}
\Theta^{\prime} & =y^{\prime} \partial \Delta_{y}-4 a^{2} y^{3} y^{\prime} \sinh ^{2} \theta \Delta_{\theta}-a^{2} y^{4} \theta^{\prime} \partial\left(\sinh ^{2} \theta \Delta_{\theta}\right), \\
\frac{T^{\prime}}{T} & =\frac{y^{\prime} \partial \Delta_{y}}{\Delta_{y}}+\frac{\theta^{\prime} \partial\left(\sinh ^{2} \theta \Delta_{\theta}\right)}{\left(\sinh ^{2} \theta \Delta_{\theta}\right)} \\
\partial \Delta_{y} & =-4 a^{2} y^{3}+2\left(a^{2}-1\right) y-2 d m y^{d-1} \\
\partial \Delta_{\theta} & =a^{2} \sinh (2 \theta) \\
\partial\left(\sinh ^{2} \theta \Delta_{\theta}\right) & =\sinh (2 \theta)\left(1+a^{2} \cosh (2 \theta)\right)
\end{aligned}
$$

and

$$
\begin{aligned}
& \frac{\partial L^{2}}{\partial y}=\frac{\partial}{\partial y}\left(\Theta+T \phi^{\prime 2}\right)=\left(1+\frac{\sinh ^{2} \theta \Delta_{\theta}}{\Xi^{2}} \phi^{\prime 2}\right) \partial \Delta_{y}-4 a^{2} y^{3} \sinh ^{2} \theta \Delta_{\theta}, \\
& \frac{\partial L^{2}}{\partial \theta}=\frac{\partial}{\partial \theta}\left(\Theta+T \phi^{\prime 2}\right)=\left(-a^{2} y^{4}+\frac{\Delta_{y}}{\Xi^{2}} \phi^{\prime 2}\right) \partial\left(\sinh ^{2} \theta \Delta_{\theta}\right)
\end{aligned}
$$


In the matrix form the equations are

$$
\left[\begin{array}{ccc}
1 & & -T y^{\prime} \phi^{\prime} / L^{2} \\
& 1 & -T \theta^{\prime} \phi^{\prime} / L^{2} \\
& & 1-T \phi^{\prime 2} / L^{2}
\end{array}\right]\left[\begin{array}{c}
y^{\prime \prime} \\
\theta^{\prime \prime} \\
\phi^{\prime \prime}
\end{array}\right]=\left[\begin{array}{c}
y^{\prime} A+y^{\prime} B_{y}+C_{y} \\
\theta^{\prime} A+\theta^{\prime} B_{\theta}+C_{\theta} \\
\phi^{\prime} A+\phi^{\prime} B_{\phi}
\end{array}\right]
$$

By multiplying the inverse matrix, these equations can be solved for the second derivative terms in the same way as the spherical case

$$
\left[\begin{array}{c}
y^{\prime \prime} \\
\theta^{\prime \prime} \\
\phi^{\prime \prime}
\end{array}\right]=\frac{1}{1-T \phi^{\prime 2} / L^{2}}\left[\begin{array}{ccc}
1-T \phi^{\prime 2} / L^{2} & 0 & T y^{\prime} \phi^{\prime} / L^{2} \\
0 & 1-T \phi^{\prime 2} / L^{2} & T \theta^{\prime} \phi^{\prime} / L^{2} \\
0 & 0 & 1
\end{array}\right]\left[\begin{array}{c}
y^{\prime} A+y^{\prime} B_{y}+C_{y} \\
\theta^{\prime} A+\theta^{\prime} B_{\theta}+C_{\theta} \\
\phi^{\prime} A+\phi^{\prime} B_{\phi}
\end{array}\right],
$$

where the coefficients are replaced with 49 .

Boundary condition The boundary condition is by the Neumann boudary condition $d \theta / d y=d \phi / d y=0$ and by the gauge condition $y^{\prime 2} / \Delta_{y}+\theta^{\prime 2} / \Delta_{\theta}=1$ at $y=0$,

$$
1=y^{\prime 2}+\frac{\theta^{\prime 2}}{1+a^{2} \cosh ^{2} \theta_{0}}, \quad \therefore\left(y, \theta, \phi, y^{\prime}, \theta^{\prime}, \phi^{\prime}\right) \stackrel{y \rightarrow 0}{\rightarrow}\left(0, \theta_{0}, 0,1,0,0\right) .
$$

The results for 4-dimension are plotted in the following figures. Figure 10 represents the mass dependence. For example, the location of the horizon is $y_{\mathrm{h}}=0.53$ for $m=5$ and $y_{\mathrm{h}}=0.37$ for $m=25$. The angular momentum dependence is plotted in Figure 11 .

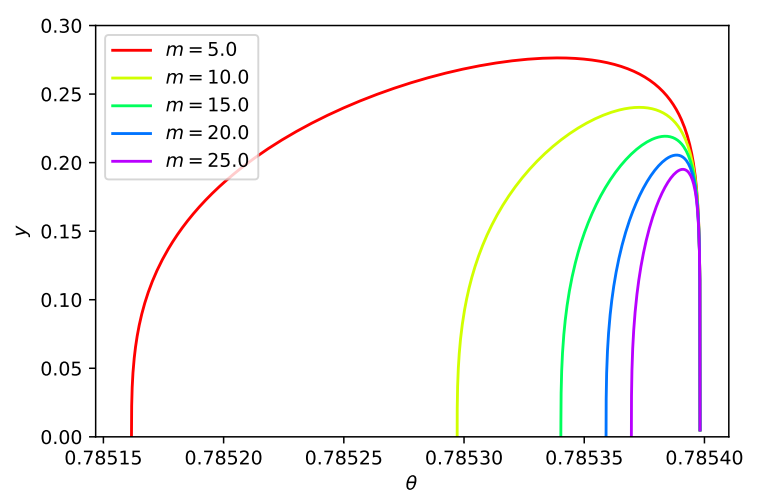

Figure 10: Mass dependence of the string em- Figure 11: Angular momentum dependence of bedding $\left(\mathbb{H}, \theta_{0}=\pi / 4\right)$

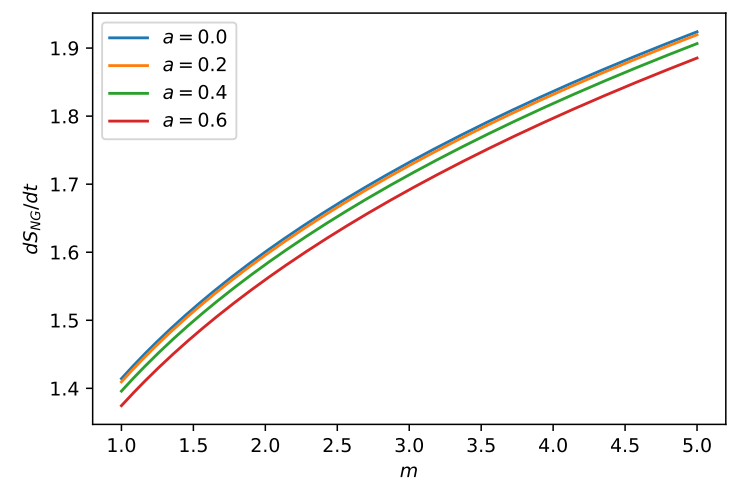

Figure 12: Mass and action growth for various angular momentums $(\mathbb{H})$

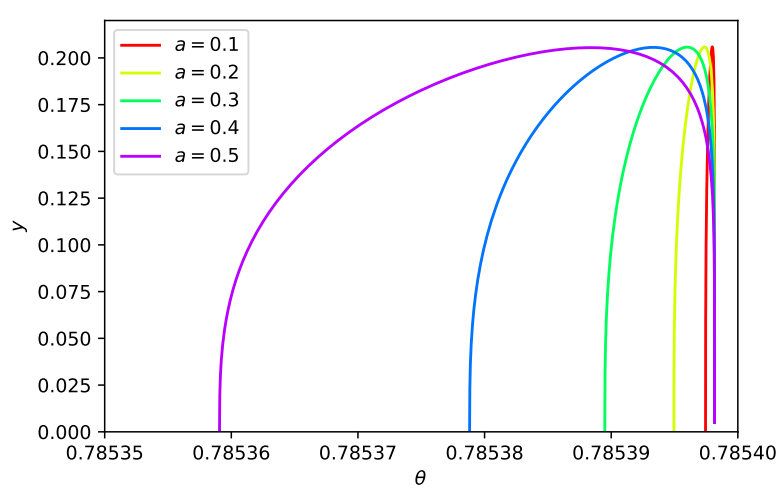

the string embedding $(\mathbb{H})$ 
Comparing Figure 7 and Figure 10 for mass dependence and Figure 8 and Figure 11 for angular momentum dependence, we can see the string is more affected in the hyperbolic case.

WDW action For $\theta_{0}=0$ the action is

$$
\frac{1}{T_{\mathrm{str}}} \frac{d S_{\mathrm{NG}}}{d t}=\int_{y_{\mathrm{h}}}^{\infty} \frac{d y}{y^{2}}=\frac{1}{y_{\mathrm{h}}}=: x,
$$

where $y_{\mathrm{h}}$ is determined by

$$
\Delta_{y}\left(y_{\mathrm{h}}\right)=\left(1+a^{2} y_{\mathrm{h}}^{2}\right)\left(1-y_{\mathrm{h}}^{2}\right)-2 m y_{\mathrm{h}}^{d}=0 .
$$

For $d=4$, by solving $x^{4}-\left(1-a^{2}\right) x^{2}-\left(2 m+a^{2}\right)=0,(x>0)$,

$$
\frac{1}{T_{\mathrm{str}}} \frac{d S_{\mathrm{NG}}}{d t}=\left(\frac{\left(1-a^{2}\right)+\sqrt{\left(1-a^{2}\right)^{2}+4\left(2 m+a^{2}\right)}}{2}\right)^{1 / 2} \text {. }
$$

The mass dependence for different angular momentums are plotted in Figure 12 .

\subsection{Toroidal case}

The induced metric of the toroidal rotating black hole is, if the spherical part is zero,

$$
\left.d s_{\mathrm{KAT}}^{2}\right|_{\text {ind }}=-\left(N^{2}-\frac{\Sigma^{2} \omega^{2}}{\rho^{2}}\right) d \tau^{2}+\left(\frac{\rho^{2}}{\Delta_{r}} r^{\prime 2}+\frac{\rho^{2}}{\Delta_{\theta}} \theta^{\prime 2}+\frac{\Sigma^{2}}{\rho^{2}} \phi^{\prime 2}\right) d \sigma^{2}-2 \frac{\omega \Sigma^{2} \phi^{\prime}}{\rho^{2}} d \tau d \sigma .
$$

In the above we used

$$
\begin{aligned}
& \rho^{2}:=r^{2}+a^{2} \theta^{2}, \quad \Delta_{\theta}:=1+a^{2} \theta^{4}, \quad \Delta_{r}:=a^{2}-2 m+r^{4}, \\
& \Sigma^{2}:=r^{4} \Delta_{\theta}-a^{2} \theta^{4} \Delta_{r}, \quad \omega:=a \frac{\Delta_{r} \theta^{2}+r^{2} \Delta_{\theta}}{\Sigma^{2}}, \quad N^{2}=\frac{\rho^{2} \Delta_{\theta} \Delta_{r}}{\Sigma^{2}} .
\end{aligned}
$$

The action is

$$
\frac{1}{T_{\mathrm{str}}} \frac{d S_{\mathrm{NG}}}{d t}=\int d \sigma \mathcal{L}_{\mathbb{T}}, \quad \mathcal{L}_{\mathbb{T}}=\sqrt{\left(\frac{\rho^{4}}{\Sigma^{2}} \Delta_{\theta}-\frac{\omega^{2} \Sigma^{2}}{\Delta_{r}}\right) r^{\prime 2}+\left(\frac{\rho^{4}}{\Sigma^{2}} \Delta_{r}-\frac{\omega^{2} \Sigma^{2}}{\Delta_{\theta}}\right) \theta^{\prime 2}+\Delta_{r} \Delta_{\theta} \phi^{\prime 2}} .
$$

where the first and the second terms have the common factor

$$
\rho^{4} \Delta_{r} \Delta_{\theta}-\omega^{2} \Sigma^{4}=\left(r^{2}+a^{2} \theta^{2}\right)^{2} \Delta_{r} \Delta_{\theta}-a^{2}\left(\theta^{2} \Delta_{r}+r^{2} \Delta_{\theta}\right)^{2}=\left(\Delta_{r}-a^{2} \Delta_{\theta}\right) \Sigma^{2} .
$$

Therefore,

$$
\mathcal{L}_{\mathbb{T}}=\sqrt{\frac{\Delta_{r}-a^{2} \Delta_{\theta}}{\Delta_{r}} r^{\prime 2}+\frac{\Delta_{r}-a^{2} \Delta_{\theta}}{\Delta_{\theta}} \theta^{\prime 2}+\Delta_{r} \Delta_{\theta} \phi^{\prime 2}}
$$

Changing variables by $y=1 / r$,

$$
\begin{aligned}
\frac{1}{T_{\mathrm{str}}} \frac{d S_{\mathrm{NG}}}{d t} & =\int d \sigma \mathcal{L}_{\mathbb{T}}, \quad \mathcal{L}_{\mathbb{T}}=\frac{L}{y^{2}}, \\
L & :=\sqrt{\left(\Delta_{y}-a^{2} y^{4} \Delta_{\theta}\right)\left(\frac{y^{\prime 2}}{\Delta_{y}}+\frac{\theta^{\prime 2}}{\Delta_{\theta}}\right)+\Delta_{y} \Delta_{\theta} \phi^{\prime 2}} .
\end{aligned}
$$

It is convenience to define the following:

$$
\begin{aligned}
& L=\sqrt{\Theta\left(\frac{y^{\prime 2}}{\Delta_{y}}+\frac{\theta^{\prime 2}}{\Delta_{\theta}}\right)+T \phi^{\prime 2}}, \\
& \Theta=\Delta_{y}-a^{2} y^{4} \Delta_{\theta}, \quad T=\Delta_{y} \Delta_{\theta}, \\
& \Delta_{\theta}=1+a^{2} \theta^{4}, \quad \Delta_{y}=a^{2} y^{4}-2 m y^{3}+1 .
\end{aligned}
$$


The equations of motion are

$$
\begin{aligned}
& y^{\prime \prime}-y^{\prime} \frac{d}{d \sigma} \log L+y^{\prime} \frac{d}{d \sigma} \log \left(\frac{\Theta}{y^{2} \Delta_{y}}\right)+\frac{\Delta_{y}}{\Theta}\left(\frac{2 L^{2}}{y}-\frac{1}{2} \frac{\partial L^{2}}{\partial y}\right)=0, \\
& \theta^{\prime \prime}-\theta^{\prime} \frac{d}{d \sigma} \log L+\theta^{\prime} \frac{d}{d \sigma} \log \left(\frac{\Theta}{y^{2} \Delta_{\theta}}\right)-\frac{\Delta_{\theta}}{2 \Theta} \frac{\partial L^{2}}{\partial \theta}=0 \\
& \phi^{\prime \prime}-\phi^{\prime} \frac{d}{d \sigma} \log L+\phi^{\prime} \frac{d}{d \sigma} \log \left(\frac{T}{y^{2}}\right)=0 .
\end{aligned}
$$

In the matrix notation the equations are the same form as before

$$
\left[\begin{array}{l}
y^{\prime \prime} \\
\theta^{\prime \prime} \\
\phi^{\prime \prime}
\end{array}\right]=\frac{1}{1-T \phi^{\prime 2} / L^{2}}\left[\begin{array}{ccc}
1-T \phi^{\prime 2} / L^{2} & 0 & T y^{\prime} \phi^{\prime} / L^{2} \\
0 & 1-T \phi^{\prime 2} / L^{2} & T \theta^{\prime} \phi^{\prime} / L^{2} \\
0 & 0 & 1
\end{array}\right]\left[\begin{array}{c}
y^{\prime} A+y^{\prime} B_{y}+C_{y} \\
\theta^{\prime} A+\theta^{\prime} B_{\theta}+C_{\theta} \\
\phi^{\prime} A+\phi^{\prime} B_{\phi}
\end{array}\right]
$$

where the coefficients are replaced with

$$
\begin{aligned}
A & :=\frac{1}{2 L^{2}}\left(y^{\prime} \partial \Delta_{y}-4 a^{2} y^{3} y^{\prime} \Delta_{\theta}-a^{2} y^{4} \theta^{\prime} \partial \Delta_{\theta}+T^{\prime} \phi^{\prime 2}\right), \\
B_{y} & :=\frac{2 y^{\prime}}{y}-\frac{\Theta^{\prime}}{\Theta}+\frac{y^{\prime} \partial \Delta_{y}}{\Delta_{y}}, B_{\theta}:=\frac{2 y^{\prime}}{y}-\frac{\Theta^{\prime}}{\Theta}+\frac{\theta^{\prime} \partial \Delta_{\theta}}{\Delta_{\theta}}, B_{\phi}:=\frac{2 y^{\prime}}{y}-\frac{T^{\prime}}{T}, \\
C_{y} & :=-\frac{\Delta_{y}}{\Theta}\left(\frac{2 L^{2}}{y}-\frac{1}{2} \frac{\partial L^{2}}{\partial y}\right), \quad C_{\theta}:=\frac{\Delta_{\theta}}{2 \Theta} \frac{\partial L^{2}}{\partial \theta} .
\end{aligned}
$$

In the above

$$
\begin{aligned}
\Theta^{\prime} & =y^{\prime} \partial \Delta_{y}-4 a^{2} y^{3} y^{\prime} \Delta_{\theta}-a^{2} y^{4} \theta^{\prime} \partial \Delta_{\theta}, \\
\frac{T^{\prime}}{T} & =\frac{y^{\prime} \partial \Delta_{y}}{\Delta_{y}}+\frac{\theta^{\prime} \partial \Delta_{\theta}}{\Delta_{\theta}}, \\
\partial \Delta_{y} & =4 a^{2} y^{3}-6 m y^{2}, \\
\partial \Delta_{\theta} & =4 a^{2} \theta^{3},
\end{aligned}
$$

and

$$
\begin{aligned}
& \frac{\partial L^{2}}{\partial y}=\frac{\partial}{\partial y}\left(\Theta+T \phi^{\prime 2}\right)=\left(1+\Delta_{\theta} \phi^{2}\right) \partial \Delta_{y}-4 a^{2} y^{3} \Delta_{\theta}, \\
& \frac{\partial L^{2}}{\partial \theta}=\frac{\partial}{\partial \theta}\left(\Theta+T \phi^{\prime 2}\right)=\left(-a^{2} y^{4}+\Delta_{y} \phi^{\prime 2}\right) \partial \Delta_{\theta} .
\end{aligned}
$$

Boundary condition The boundary condition is by the Neumann boudary condition $d \theta / d y=d \phi / d y=0$ and by the gauge condition $y^{\prime 2} / \Delta_{y}+\theta^{\prime 2} / \Delta_{\theta}=1$ at $y=0$,

$$
1=y^{\prime 2}+\frac{\theta^{\prime 2}}{1+a^{2} \theta_{0}^{4}}, \quad \therefore\left(y, \theta, \phi, y^{\prime}, \theta^{\prime}, \phi^{\prime}\right) \stackrel{y \rightarrow 0}{\rightarrow}\left(0, \theta_{0}, 0,1,0,0\right) .
$$

Figure 13 represents the mass dependence. For example, $y_{\mathrm{h}}=0.47$ for $m=5$ and $y_{\mathrm{h}}=0.27$ for $m=25$. The angular momentum dependence is plotted in Figure 14 .

For $\theta_{0}=0$ the action growth is

$$
\frac{d S_{\mathrm{NG}}}{d t}=\frac{1}{2 \pi \alpha^{\prime}} \int_{y_{\mathrm{h}}}^{\infty} \frac{d y}{y^{2}} \sqrt{1-\frac{a^{2} y^{4}}{\Delta_{y}}}=\frac{1}{2 \pi \alpha^{\prime}} \int_{y_{\mathrm{h}}}^{\infty} \frac{d y}{y^{2}} \sqrt{\frac{1-2 m y^{3}}{1-2 m y^{3}+a^{2} y^{4}}} .
$$

In the square root the numerator is less than the denominator for non zero $a$. Then it cannot penetrate the horizon for $a \neq 0$.

Comparing Figure 7 and Figure 13 for mass dependence and Figure 8 and Figure 14 for angular momentum dependence, we can see the string is tend to receive more effect than sphere and hyperbolic cases as seen for small masses or large angular momentum. 


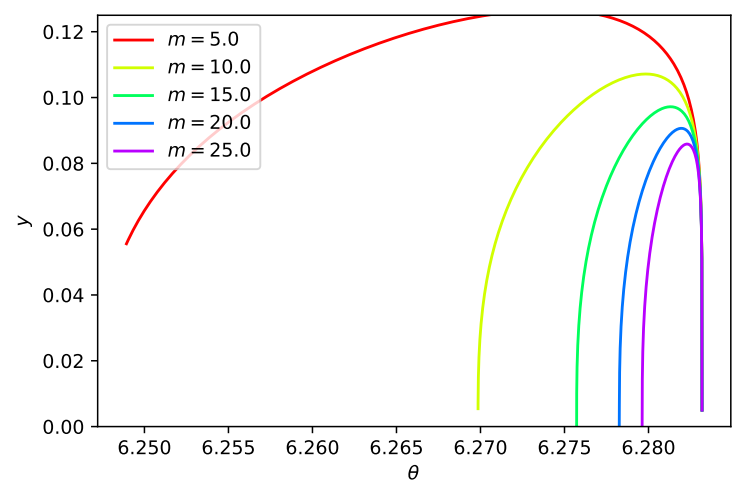

Figure 13: Mass dependence of the string em- Figure 14: Angular momentum dependence of bedding $(\mathbb{T})$

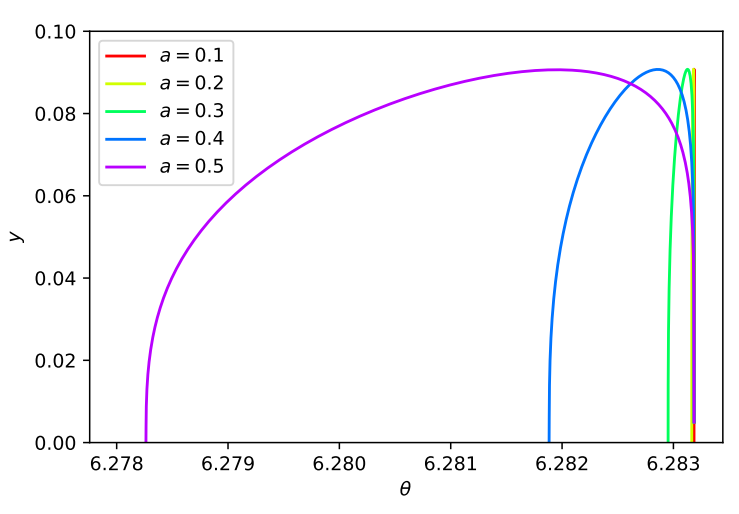

the string embedding $(\mathbb{T})$

\section{Discussion}

In this paper we found the behavior of the fundamental strings in black hole spacetime with three different horizon structures. In (1) the curvature parameter $k$ distinguishes these cases: $k=1$ is the spherical horizon case, $k=-1$ is the hyperbolic case and $k=0$ is the toroidal horizon case.

First in Section 2 we studied the static black holes and found that the constant solution $\theta^{\prime}=0$ is only able to approach the horizon for all cases $(k= \pm 1,0)$. The growth of the Nambu-Goto (NG) action on the Wheeler DeWitt (WDW) is proportional to the horizon radius. Only for the hyperbolic black holes, there is a non-trivial contribution even for zero-mass, where the metric function $g_{-1}(r)$ has a zero point $r_{\mathrm{h}}=1$.

For rotating topological black holes, the behavior of the strings are summarized in Figure 15. In this figure, the dashed lines represent the strings which bend and do not extend to the interior of the horizon. As we found in the calculation, such strings approach the AdS boundary at non-zero altitude angle $\theta \neq 0$ for the spherical and the hyperbolic cases. In the left and the middle panels the dotted lines are strings which attach the boundary at $\theta=0$. It is the only case where the string can penetrate the horizon of the black holes. A remarkable point is that there is not such a solution for toroidal case (the right panel). For the toroidal case, the string can penetrate the horizon only if the black hole is not rotating.

For spherical $k=1$ and hyperbolic $k=-1$ cases, there exist solutions which penetrate the horizon if the strings attach the boundary at $\theta_{0}=0$. In these cases we obtained the WDW action. Figure 9 and Figure 12 say the growth of the action is an increasing function of mass and the angular momentum has the negative contribution to it.

For toroidal case, $k=0$, we found that there exists the string solution which penetrates the horizon only for zero angular momentum $a=0$. In this case the effect of the fundamental string to the growth of the action is given in Figure 2 and Figure 3 $(k=0)$ in Section 2. This is also an increasing function of mass. However, as mentioned in Introduction and the begging of Section 2.1, in the static situation this does not have an interpretation for the drag force. Moreover, since the string cannot exist in the horizon in this case, the string is not a good probe to capture the property of complexity growth for toroidal black holes.

Let us see the holographic interpretation of these spacetimes. In the holographic gauge theory, the fundamental string we considered in this paper is interpreted as a nonlocal gauge theory object, "a Wilson loop." The edge of the string at the AdS boundary is a test particle with infinite mass. Therefore we conclude that for the spherical and 

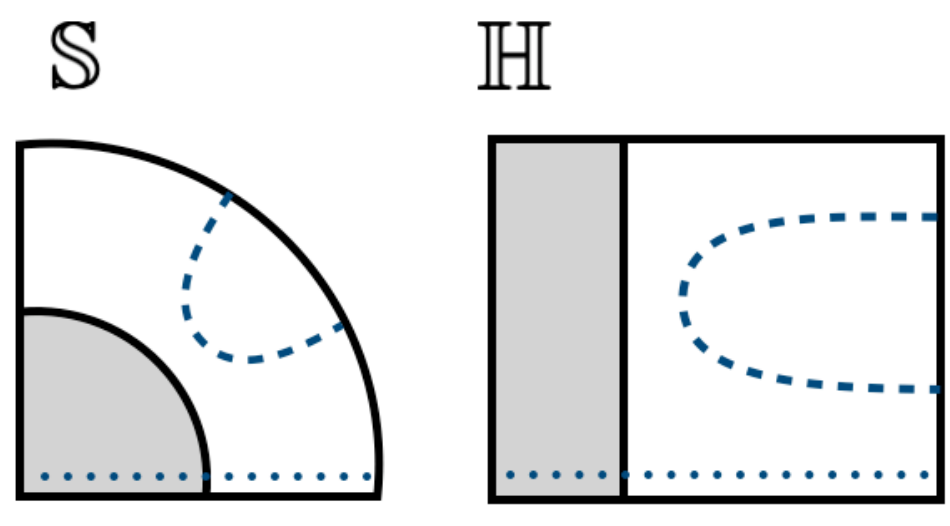
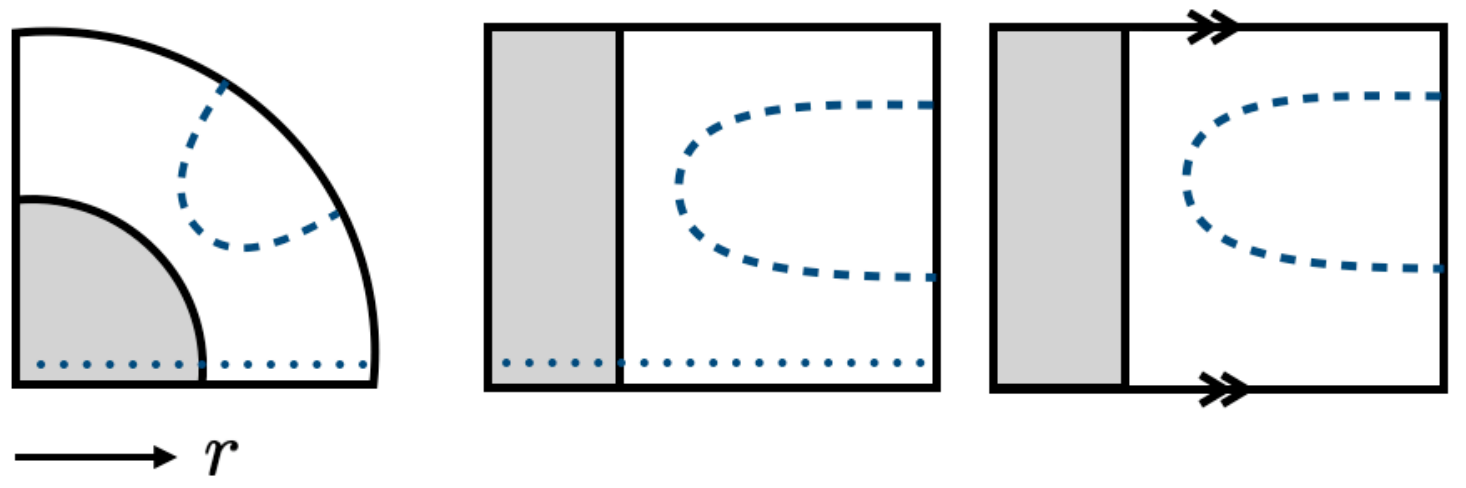

Figure 15: Sketch of string behavior in black hole spacetime for different horizon structures: For each case the shaded region is the inside of the horizon. The horizontal direction is the radial direction $r$ and the vertical direction is $\theta$. The right edge or the outer edge is the AdS boundary for each case. The dotted line which starts with $\theta=0$ is the only case where the string can penetrates the horizon. The dashed line starts with $\theta \neq 0$ and such a string does not enter the horizon. Left: For spherical case $(\mathbb{S})$ the region of $\theta$ is $0 \leq \theta \leq \pi$. Middle: For hyperbolic case $(\mathbb{H})$ the region of $\theta$ is $0 \leq \theta$. Right: For Toroidal case $(\mathbb{T})$ the region of $\theta$ is $0 \leq \theta \leq 1$ and the top side and the bottom side are identified.

the hyperbolic horizon black holes there is a possibility that there are two particles or one particle on the AdS boundary while for the toroidal horizon case, there are always two particles on the dual gauge theory which are edges of one fundamental string.

Let us also discuss a condition which any physical system must satisfy. For the rate of computation the system may satisfy "Lloyd's bound," [161, 162, 163]:

$$
\frac{d S}{d t} \leq \frac{2}{\pi \hbar}(M-\Omega J)
$$

where $\Omega$ is the angular velocity and $J$ is the angular momentum. On the lefthand side of $(73), S$ is the total action which now consists of $S=S_{\text {bulk }}+S_{\mathrm{NG}}$, where $S_{\text {bulk }}$ includes the Einstein-Hilbert term and the some boundary terms. On the righthand side of the inequality (73) $M$ is the total mass or energy of the system. In our case, not only the black hole mass, we have to take into account the mass of the string. However, the string stretches towards the infinity of the space and it has the infinite length. Therefore in our calculation this inequality is automatically satisfied.

Finally from the result of this paper we can say that the angular momentum has the effect for decreasing the rate of the complexity growth for all cases $(\mathbb{S}, \mathbb{H}$ and $\mathbb{T}$ ). This outcome is consistent with the above comment for Lloyd's bound which says the the upper bound decreases for rotating black holes. It may be counterintuitive that a dynamical system has the less growth rate of complexity but the same phenomena was also found in [67, 68, where we studied a moving fundamental string in AdS black hole spacetime. Furthermore, the growth rate of the complexity was smaller when the relative velocity between the string and the angular momentum of the black holes are small. 


\section{Acknnowledgments}

I would like to thank Sung-Soo Kim and Satoshi Yamaguchi for helpful discussion.

\section{References}

[1] J. M. Maldacena, "The Large N limit of superconformal field theories and supergravity," Int. J. Theor. Phys. 38 (1999) 1113-1133, arXiv:hep-th/9711200 [hep-th]. [Adv. Theor. Math. Phys.2,231(1998)].

[2] E. Witten, "Anti-de Sitter space and holography," Adv.Theor.Math.Phys. 2 (1998) 253-291, arXiv:hep-th/9802150 [hep-th].

[3] A. M. Awad and C. V. Johnson, "Higher dimensional Kerr - AdS black holes and the AdS / CFT correspondence," Phys. Rev. D63 (2001) 124023. arXiv:hep-th/0008211 [hep-th].

[4] S. S. Gubser, "Drag force in AdS/CFT," Phys. Rev. D74 (2006) 126005, arXiv:hep-th/0605182 [hep-th].

[5] A. Nata Atmaja and K. Schalm, "Anisotropic Drag Force from 4D Kerr-AdS Black Holes," JHEP 04 (2011) 070, arXiv: 1012.3800 [hep-th].

[6] C. P. Herzog, A. Karch, P. Kovtun, C. Kozcaz, and L. G. Yaffe, "Energy loss of a heavy quark moving through $\mathrm{N}=4$ supersymmetric Yang-Mills plasma," JHEP 07 (2006) 013, arXiv:hep-th/0605158 [hep-th].

[7] J. Casalderrey-Solana and D. Teaney, "Heavy quark diffusion in strongly coupled N=4 Yang-Mills," Phys. Rev. D74 (2006) 085012, arXiv:hep-ph/0605199 [hep-ph].

[8] H. Liu, K. Rajagopal, and U. A. Wiedemann, "Calculating the jet quenching parameter from AdS/CFT," Phys. Rev. Lett. 97 (2006) 182301, arXiv:hep-ph/0605178 [hep-ph].

[9] K. Bitaghsir Fadafan, H. Liu, K. Rajagopal, and U. A. Wiedemann, "Stirring Strongly Coupled Plasma," Eur. Phys. J. C61 (2009) 553-567, arXiv:0809.2869 [hep-ph].

[10] K. B. Fadafan and H. Soltanpanahi, "Energy loss in a strongly coupled anisotropic plasma," JHEP 10 (2012) 085, arXiv:1206.2271 [hep-th].

[11] M. Atashi, K. Bitaghsir Fadafan, and M. Farahbodnia, "Holographic energy loss in non-relativistic backgrounds," Eur. Phys. J. C77 no. 3, (2017) 175. arXiv:1606.09491 [hep-th]

[12] K. Nagasaki, H. Tanida, and S. Yamaguchi, "Holographic Interface-Particle Potential," JHEP 01 (2012) 139, arXiv:1109.1927 [hep-th].

[13] Y. Sekino and L. Susskind, "Fast Scramblers," JHEP 10 (2008) 065. arXiv:0808.2096 [hep-th].

[14] S. R. Coleman, J. Preskill, and F. Wilczek, "Quantum hair on black holes," Nucl. Phys. B378 (1992) 175-246, arXiv:hep-th/9201059 [hep-th].

[15] G. Dvali, C. Gomez, D. Lust, Y. Omar, and B. Richter, "Universality of Black Hole Quantum Computing," Fortsch. Phys. 65 no. 1, (2017) 1600111, arXiv:1605.01407 [hep-th]

[16] B. Swingle, G. Bentsen, M. Schleier-Smith, and P. Hayden, "Measuring the scrambling of quantum information," Phys. Rev. A94 no. 4, (2016) 040302 arXiv:1602.06271 [quant-ph]. 
[17] T. J. Osborne, "Hamiltonian complexity," Reports on Progress in Physics 75 no. 2, 022001 .

[18] J. Watrous, "Quantum Computational Complexity," ArXiv e-prints (Apr., 2008), arXiv:0804.3401 [quant-ph].

[19] S. Arora and B. Barak, "Computational Complexity: A Modern Approach". Cambridge University Press, New York, NY, USA, 1st ed., 2009.

[20] C. Moore and S. Mertens, "The Nature of Computation". Oxford University Press, Inc., New York, NY, USA, 2011.

[21] L. Susskind, "Entanglement is not enough," Fortsch. Phys. 64 (2016) 49-71, arXiv:1411.0690 [hep-th].

[22] L. Susskind, "Computational Complexity and Black Hole Horizons," Fortsch. Phys. 64 (2016) 24-43, arXiv:1403.5695 [hep-th].

[23] P. Caputa, N. Kundu, M. Miyaji, T. Takayanagi, and K. Watanabe, "Liouville Action as Path-Integral Complexity: From Continuous Tensor Networks to AdS/CFT," JHEP 11 (2017) 097, arXiv:1706.07056 [hep-th].

[24] K. Hashimoto, N. Iizuka, and S. Sugishita, "Thoughts on Holographic Complexity and its Basis-dependence," Phys. Rev. D98 no. 4, (2018) 046002, arXiv:1805.04226 [hep-th]

[25] L. Susskind, "Black Holes and Complexity Classes," arXiv:1802.02175 [hep-th].

[26] L. Susskind, "Three Lectures on Complexity and Black Holes," 2018. arXiv:1810.11563 [hep-th]

[27] A. Bhattacharyya, P. Caputa, S. R. Das, N. Kundu, M. Miyaji, and T. Takayanagi, "Path-Integral Complexity for Perturbed CFTs," arXiv:1804.01999 [hep-th]

[28] A. R. Brown and L. Susskind, "Second law of quantum complexity," Phys. Rev. D97 no. 8, (2018) 086015, arXiv:1701.01107 [hep-th].

[29] D. Carmi, S. Chapman, H. Marrochio, R. C. Myers, and S. Sugishita, "On the Time Dependence of Holographic Complexity," JHEP 11 (2017) 188, arXiv:1709.10184 [hep-th]

[30] Z.-Y. Fan and M. Guo, "Holographic complexity and thermodynamics of AdS black holes," Phys. Rev. D100 (2019) 026016, arXiv: 1903.04127 [hep-th].

[31] V. Vanchurin, "Dual Field Theories of Quantum Computation," JHEP 06 (2016) 001, arXiv: 1603.07982 [hep-th].

[32] S. Chapman, M. P. Heller, H. Marrochio, and F. Pastawski, "Towards Complexity for Quantum Field Theory States," Phys. Rev. Lett. 120 no. 12, (2018) 121602, arXiv:1707.08582 [hep-th].

[33] R. Jefferson and R. C. Myers, "Circuit complexity in quantum field theory," JHEP 10 (2017) 107, arXiv: 1707.08570 [hep-th].

[34] J. Couch, S. Eccles, W. Fischler, and M.-L. Xiao, "Holographic complexity and noncommutative gauge theory," JHEP 03 (2018) 108, arXiv: 1710.07833 [hep-th].

[35] A. Bhattacharyya, A. Shekar, and A. Sinha, "Circuit complexity in interacting QFTs and RG flows," JHEP 10 (2018) 140, arXiv:1808.03105 [hep-th].

[36] T. Ali, A. Bhattacharyya, S. Shajidul Haque, E. H. Kim, and N. Moynihan, "Time Evolution of Complexity: A Critique of Three Methods," JHEP 04 (2019) 087, arXiv:1810.02734 [hep-th]. 
[37] J. Jiang, J. Shan, and J. Yang, "Circuit complexity for free Fermion with a mass quench," arXiv:1810.00537 [hep-th]

[38] M. Guo, J. Hernandez, R. C. Myers, and S.-M. Ruan, "Circuit Complexity for Coherent States," JHEP 10 (2018) 011, arXiv:1807.07677 [hep-th].

[39] R. Q. Yang, Y. S. An, C. Niu, C. Y. Zhang, and K. Y. Kim, "Axiomatic complexity in quantum field theory and its applications," arXiv:1803.01797 [hep-th].

[40] L. Hackl and R. C. Myers, "Circuit complexity for free fermions," arXiv:1803.10638 [hep-th]

[41] R. Khan, C. Krishnan, and S. Sharma, "Circuit Complexity in Fermionic Field Theory," arXiv:1801.07620 [hep-th].

[42] R.-Q. Yang, Y.-S. An, C. Niu, C.-Y. Zhang, and K.-Y. Kim, "More on complexity of operators in quantum field theory," JHEP 03 (2019) 161 arXiv:1809.06678 [hep-th]

[43] S. Chapman and H. Z. Chen, "Complexity for Charged Thermofield Double States," arXiv:1910.07508 [hep-th].

[44] R.-Q. Yang, H.-S. Jeong, C. Niu, and K.-Y. Kim, "Complexity of Holographic Superconductors," JHEP 04 (2019) 146, arXiv: 1902.07586 [hep-th].

[45] G. Jafari, A. Naseh, and H. Zolfi, "Path Integral Optimization for $T \bar{T}$ Deformation," arXiv:1909.02357 [hep-th]

[46] R.-Q. Yang, Y.-S. An, C. Niu, C.-Y. Zhang, and K.-Y. Kim, "To be unitary-invariant or not?: a simple but non-trivial proposal for the complexity between states in quantum mechanics/field theory," arXiv:1906.02063 [hep-th].

[47] M. Sinamuli and R. B. Mann, "Holographic Complexity and Charged Scalar Fields," Phys. Rev. D99 no. 10, (2019) 106013, arXiv:1902.01912 [hep-th].

[48] H. Guo, X.-M. Kuang, and B. Wang, "Holographic entanglement entropy and complexity in Stückelberg superconductor," Phys. Lett. B797 (2019) 134879. arXiv:1902.07945 [hep-th]

[49] M. A. Nielsen, "A geometric approach to quantum circuit lower bounds," eprint arXiv:quant-ph/0502070 (Feb., 2005), quant-ph/0502070.

[50] M. A. Nielsen, M. R. Dowling, M. Gu, and A. C. Doherty, "Quantum Computation as Geometry," Science 311 (Feb., 2006) 1133-1135. quant-ph/0603161.

[51] M. R. Dowling and M. A. Nielsen, "The geometry of quantum computation," eprint arXiv:quant-ph/0701004 (Dec., 2007), quant-ph/0701004.

[52] A. R. Brown, L. Susskind, and Y. Zhao, "Quantum Complexity and Negative Curvature," Phys. Rev. D95 no. 4, (2017) 045010, arXiv: 1608.02612 [hep-th].

[53] R. Abt, J. Erdmenger, H. Hinrichsen, C. M. Melby-Thompson, R. Meyer, C. Northe, and I. A. Reyes, "Topological Complexity in $\mathrm{AdS}_{3} / \mathrm{CFT}_{2}$," Fortsch. Phys. 66 no. 6, (2018) 1800034, arXiv:1710.01327 [hep-th]

[54] N. Bao, C. Cao, S. M. Carroll, and A. Chatwin-Davies, "De Sitter Space as a Tensor Network: Cosmic No-Hair, Complementarity, and Complexity," Phys. Rev. D96 no. 12, (2017) 123536, arXiv:1709.03513 [hep-th].

[55] B. Czech, "Einstein Equations from Varying Complexity," Phys. Rev. Lett. 120 no. 3, (2018) 031601, arXiv:1706.00965 [hep-th]. 
[56] S. Chapman, J. Eisert, L. Hackl, M. P. Heller, R. Jefferson, H. Marrochio, and R. C. Myers, "Complexity and entanglement for thermofield double states," arXiv:1810.05151 [hep-th]

[57] M. Doroudiani, A. Naseh, and R. Pirmoradian, "Complexity for Charged Thermofield Double States," arXiv:1910.08806 [hep-th].

[58] H. W. Lin and L. Susskind, "Complexity Geometry and Schwarzian Dynamics," arXiv:1911.02603 [hep-th]

[59] J. Jiang and X. Liu, "Circuit Complexity for Fermionic Thermofield Double states," Phys. Rev. D99 no. 2, (2019) 026011, arXiv:1812.00193 [hep-th].

[60] A. R. Brown and L. Susskind, "The Complexity Geometry of a Single Qubit," arXiv:1903.12621 [hep-th]

[61] A. Bernamonti, F. Galli, J. Hernandez, R. C. Myers, S.-M. Ruan, and J. Simon, "First Law of Holographic Complexity," Phys. Rev. Lett. 123 no. 8, (2019) 081601, arXiv:1903.04511 [hep-th].

[62] M. Moosa, "Evolution of Complexity Following a Global Quench," JHEP 03 (2018) 031, arXiv:1711.02668 [hep-th].

[63] F. J. G. Abad, M. Kulaxizi, and A. Parnachev, "On Complexity of Holographic Flavors," JHEP 01 (2018) 127, arXiv: 1705.08424 [hep-th].

[64] Z. Fu, A. Maloney, D. Marolf, H. Maxfield, and Z. Wang, "Holographic complexity is nonlocal," JHEP 02 (2018) 072, arXiv:1801.01137 [hep-th].

[65] T. Numasawa, "Holographic Complexity for disentangled states," arXiv:1811.03597 [hep-th]

[66] D. S. Ageev and I. Y. Arefeva, "Holography and nonlocal operators for the BTZ black hole with nonzero angular momentum," Theor. Math. Phys. 180 (2014) 881-893, arXiv:1402.6937 [hep-th]. [Teor. Mat. Fiz.180,no.2,147(2014)].

[67] K. Nagasaki, "Complexity of $\mathrm{AdS}_{5}$ black holes with a rotating string," Phys. Rev. D96 no. 12, (2017) 126018, arXiv:1707.08376 [hep-th].

[68] K. Nagasaki, "Complexity growth of rotating black holes with a probe string," Phys. Rev. D98 no. 12, (2018) 126014, arXiv:1807.01088 [hep-th].

[69] A. R. Brown, D. A. Roberts, L. Susskind, B. Swingle, and Y. Zhao, "Holographic Complexity Equals Bulk Action?," Phys. Rev. Lett. 116 no. 19, (2016) 191301, arXiv:1509.07876 [hep-th].

[70] A. R. Brown, D. A. Roberts, L. Susskind, B. Swingle, and Y. Zhao, "Complexity, action, and black holes," Phys. Rev. D93 no. 8, (2016) 086006 arXiv:1512.04993 [hep-th]

[71] M. Alishahiha, "Holographic Complexity," Phys. Rev. D92 no. 12, (2015) 126009 , arXiv: 1509.06614 [hep-th].

[72] S. Chapman, H. Marrochio, and R. C. Myers, "Complexity of Formation in Holography," JHEP 01 (2017) 062, arXiv: 1610.08063 [hep-th].

[73] J. Tao, P. Wang, and H. Yang, "Testing holographic conjectures of complexity with Born-Infeld black holes," Eur. Phys. J. C77 no. 12, (2017) 817, arXiv:1703.06297 [hep-th]

[74] J. Jiang and X.-W. Li, "Modified "complexity equals action" conjecture," Phys. Rev. D100 no. 6, (2019) 066026, arXiv:1903.05476 [hep-th].

[75] J. Jiang and B.-X. Ge, "Investigating two counting methods of the holographic complexity," Phys. Rev. D99 no. 12, (2019) 126006, arXiv:1905.08447 [hep-th]. 
[76] Z.-Y. Fan and M. Guo, "On the Noether charge and the gravity duals of quantum complexity," JHEP 08 (2018) 031, arXiv:1805.03796 [hep-th]

[77] J. Preskill, "Do black holes destroy information?," in "International Symposium on Black holes, Membranes, Wormholes and Superstrings Woodlands, Texas, January 16-18, 1992", pp. 22-39. 1992. arXiv:hep-th/9209058 [hep-th].

[78] S. B. Giddings, "Comments on information loss and remnants," Phys. Rev. D49 (1994) 4078-4088, arXiv:hep-th/9310101 [hep-th].

[79] J. G. Russo, "The Information problem in black hole evaporation: Old and recent results," in

Beyond General Relativity. Proceedings, 2004 Spanish Relativity Meeting (ERE2004). 2007. 2005. arXiv:hep-th/0501132 [hep-th].

[80] P. Hayden and J. Preskill, "Black holes as mirrors: Quantum information in random subsystems," JHEP 09 (2007) 120, arXiv:0708.4025 [hep-th].

[81] D. R. Terno, "Black hole information problem and quantum gravity," arXiv:0909.4143 [gr-qc]. [AIP Conf. Proc.1196,284(2009)].

[82] L. Susskind, "Singularities, Firewalls, and Complementarity," arXiv:1208.3445 [hep-th].

[83] A. Almheiri, D. Marolf, J. Polchinski, and J. Sully, "Black Holes: Complementarity or Firewalls?," JHEP 02 (2013) 062, arXiv:1207.3123 [hep-th].

[84] D. Harlow and P. Hayden, "Quantum Computation vs. Firewalls," JHEP 06 (2013) 085, arXiv:1301.4504 [hep-th].

[85] J. Maldacena and L. Susskind, "Cool horizons for entangled black holes," Fortsch. Phys. 61 (2013) 781-811, arXiv:1306.0533 [hep-th].

[86] D. Stanford and L. Susskind, "Complexity and Shock Wave Geometries," Phys. Rev. D90 no. 12, (2014) 126007, arXiv:1406.2678 [hep-th]

[87] L. Susskind and Y. Zhao, "Switchbacks and the Bridge to Nowhere," arXiv: 1408.2823 [hep-th].

[88] H. Stoltenberg and A. Albrecht, "No Firewalls or Information Problem for Black Holes Entangled with Large Systems," Phys. Rev. D91 no. 2, (2015) 024004, arXiv: 1408.5179 [gr-qc].

[89] R. B. Mann, "Black Holes: Thermodynamics, Information, and Firewalls". SpringerBriefs in Physics. Springer, 2015.

[90] J. L. F. Barbon and E. Rabinovici, "Holographic complexity and spacetime singularities," JHEP 01 (2016) 084, arXiv: 1509.09291 [hep-th].

[91] L. Susskind, "The Typical-State Paradox: Diagnosing Horizons with Complexity," Fortsch. Phys. 64 (2016) 84-91, arXiv:1507.02287 [hep-th].

[92] D. Momeni, S. A. H. Mansoori, and R. Myrzakulov, "Holographic Complexity in Gauge/String Superconductors," Phys. Lett. B756 (2016) 354-357, arXiv:1601.03011 [hep-th].

[93] J. Couch, W. Fischler, and P. H. Nguyen, "Noether charge, black hole volume, and complexity," JHEP 03 (2017) 119, arXiv:1610.02038 [hep-th].

[94] D. A. Roberts and B. Yoshida, "Chaos and complexity by design," JHEP 04 (2017) 121, arXiv:1610.04903 [quant-ph].

[95] J. Polchinski, "The Black Hole Information Problem," in Proceedings, TASI 2015: Boulder, June 1-26, 2015. 2017. 
[96] Y. Zhao, "Complexity, boost symmetry, and firewalls," arXiv:1702.03957 [hep-th].

[97] D. Marolf, "The Black Hole information problem: past, present, and future," Rept. Prog. Phys. 80 no. 9, (2017) 092001, arXiv:1703.02143 [gr-qc].

[98] W. C. Gan and F. W. Shu, "Holographic complexity: A tool to probe the property of reduced fidelity susceptibility," Phys. Rev. D96 no. 2, (2017) 026008, arXiv:1702.07471 [hep-th].

[99] X. H. Ge and B. Wang, "Quantum computational complexity, Einstein's equations and accelerated expansion of the Universe," JCAP 1802 no. 02, (2018) 047, arXiv:1708.06811 [hep-th].

[100] S. A. Hosseini Mansoori and M. M. Qaemmaqami, "Complexity Growth, Butterfly Velocity and Black hole Thermodynamics," arXiv:1711.09749 [hep-th].

[101] M. Kord Zangeneh, Y. C. Ong, and B. Wang, "Entanglement Entropy and Complexity for One-Dimensional Holographic Superconductors," Phys. Lett. B771 (2017) 235-241, arXiv:1704.00557 [hep-th].

[102] S. Lloyd, "Ultimate physical limits to computation," nat 406 (Aug., 2000) quant-ph/9908043.

[103] J. L. F. Barbon and J. Martin-Garcia, "Holographic Complexity Of Cold Hyperbolic Black Holes," JHEP 11 (2015) 181, arXiv:1510.00349 [hep-th].

[104] W. J. Pan and Y. C. Huang, "Holographic complexity and action growth in massive gravities," Phys. Rev. D95 no. 12, (2017) 126013, arXiv:1612.03627 [hep-th].

[105] H. Huang, X. H. Feng, and H. Lu, "Holographic Complexity and Two Identities of Action Growth," Phys. Lett. B769 (2017) 357-361, arXiv:1611.02321 [hep-th].

[106] D. Momeni, M. Faizal, S. Bahamonde, and R. Myrzakulov, "Holographic complexity for time-dependent backgrounds," Phys. Lett. B762 (2016) 276-282, arXiv:1610.01542 [hep-th]

[107] R. G. Cai, S. M. Ruan, S. J. Wang, R. Q. Yang, and R. H. Peng, "Action growth for AdS black holes," JHEP 09 (2016) 161, arXiv: 1606.08307 [gr-qc].

[108] K. Hashimoto, N. Iizuka, and S. Sugishita, "Time evolution of complexity in Abelian gauge theories," Phys. Rev. D96 no. 12, (2017) 126001, arXiv:1707.03840 [hep-th]

[109] M. Alishahiha, A. Faraji Astaneh, A. Naseh, and M. H. Vahidinia, "On complexity for F(R) and critical gravity," JHEP 05 (2017) 009 , arXiv:1702.06796 [hep-th]

[110] A. Reynolds and S. F. Ross, "Complexity in de Sitter Space," Class. Quant. Grav. 34 no. 17, (2017) 175013, arXiv:1706.03788 [hep-th].

[111] M. M. Qaemmaqami, "Complexity growth in minimal massive 3D gravity," Phys. Rev. D97 no. 2, (2018) 026006, arXiv:1709.05894 [hep-th].

[112] R. Q. Yang, "Complexity for quantum field theory states and applications to thermofield double states," Phys. Rev. D97 no. 6, (2018) 066004. arXiv:1709.00921 [hep-th]

[113] S. Karar and S. Gangopadhyay, "Holographic complexity for Lifshitz system," Phys. Rev. D98 no. 2, (2018) 026029, arXiv:1711.10887 [hep-th].

[114] Y. G. Miao and L. Zhao, "Complexity-action duality of the shock wave geometry in a massive gravity theory," Phys. Rev. D97 no. 2, (2018) 024035 , arXiv:1708.01779 [hep-th]. 
[115] M. Ghodrati, "Complexity growth in massive gravity theories, the effects of chirality, and more," Phys. Rev. D96 no. 10, (2017) 106020, arXiv:1708.07981 [hep-th].

[116] W. D. Guo, S. W. Wei, Y. Y. Li, and Y. X. Liu, "Complexity growth rates for AdS black holes in massive gravity and $f(R)$ gravity," Eur. Phys. J. C77 no. 12, (2017) 904, arXiv:1703.10468 [gr-qc].

[117] L. Sebastiani, L. Vanzo, and S. Zerbini, "Action growth for black holes in modified gravity," Phys. Rev. D97 no. 4, (2018) 044009, arXiv:1710.05686 [hep-th].

[118] R. G. Cai, M. Sasaki, and S. J. Wang, "Action growth of charged black holes with a single horizon," Phys. Rev. D95 no. 12, (2017) 124002 , arXiv:1702.06766 [gr-qc].

[119] P. Wang, H. Yang, and S. Ying, "Action Growth in $f(R)$ Gravity," arXiv:1703.10006 [hep-th]

[120] B. Swingle and Y. Wang, "Holographic Complexity of Einstein-Maxwell-Dilaton Gravity," arXiv:1712.09826 [hep-th].

[121] P. A. Cano, R. A. Hennigar, and H. Marrochio, "Complexity Growth Rate in Lovelock Gravity," arXiv:1803.02795 [hep-th].

[122] A. R. Brown, H. Gharibyan, H. W. Lin, L. Susskind, L. Thorlacius, and Y. Zhao, "The Case of the Missing Gates: Complexity of Jackiw-Teitelboim Gravity," arXiv:1810.08741 [hep-th].

[123] H. Ghaffarnejad, M. Farsam, and E. Yaraie, "Effects of quintessence dark energy on the action growth and butterfly velocity," arXiv:1806.05735 [hep-th].

[124] S. Chakraborty and K. Parattu, "Null Boundary Terms for Lanczos-Lovelock Gravity," arXiv:1806.08823 [gr-qc].

[125] S. Chapman, H. Marrochio, and R. C. Myers, "Holographic complexity in Vaidya spacetimes. Part I," JHEP 06 (2018) 046, arXiv:1804.07410 [hep-th].

[126] S. A. Hosseini Mansoori, V. Jahnke, M. M. Qaemmaqami, and Y. D. Olivas, "Holographic complexity of anisotropic black branes," Phys. Rev. D100 no. 4, (2019) 046014, arXiv: 1808.00067 [hep-th].

[127] S. Mahapatra and P. Roy, "On the time dependence of holographic complexity in a dynamical Einstein-dilaton model," JHEP 11 (2018) 138 arXiv:1808.09917 [hep-th]

[128] A. Ovgun and K. Jusufi, "Complexity growth rates for AdS black holes with dyonic/ nonlinear charge/ stringy hair/ topological defects," arXiv:1801.09615 [gr-qc].

[129] S. Chapman, H. Marrochio, and R. C. Myers, "Holographic Complexity in Vaidya Spacetimes II," JHEP 06 (2018) 114, arXiv:1805.07262 [hep-th].

[130] R. Fareghbal and P. Karimi, "Complexity Growth in Flatland," arXiv:1806.07273 [hep-th]

[131] K. Bamba, D. Momeni, and M. Al Ajmi, "Holographic entanglement entropy, complexity, fidelity susceptibility and hierarchical UV/IR mixing problem in $\mathrm{AdS}_{2}$ /open strings," Int. J. Mod. Phys. A33 no. 17, (2018) 1850100, arXiv:1806.02209 [hep-th]

[132] R. Auzzi, S. Baiguera, M. Grassi, G. Nardelli, and N. Zenoni, "Complexity and action for warped AdS black holes," arXiv:1806.06216 [hep-th].

[133] J. Jiang, "Action growth rate for a higher curvature gravitational theory," arXiv:1810.00758 [hep-th] 
[134] H. Ghaffarnejad, E. Yaraie, and M. Farsam, "Complexity growth and shock wave geometry in AdS-Maxwell-power-Yang-Mills theory," arXiv:1806.07242 [gr-qc].

[135] X.-H. Feng and H.-S. Liu, "Holographic Complexity Growth Rate in Horndeski Theory," Eur. Phys. J. C79 no. 1, (2019) 40, arXiv:1811.03303 [hep-th].

[136] J. Jiang, "Holographic complexity in charged Vaidya black hole," Eur. Phys. J. C79 no. 2, (2019) 130, arXiv:1811.07347 [hep-th].

[137] K. Meng, "Holographic complexity of Born-Infeld black holes," arXiv:1810.02208 [hep-th]

[138] Y. S. An and R. H. Peng, "Effect of the dilaton on holographic complexity growth," Phys. Rev. D97 no. 6, (2018) 066022, arXiv:1801.03638 [hep-th].

[139] Y. Ling, Y. Liu, and C.-Y. Zhang, "Holographic Subregion Complexity in Einstein-Born-Infeld theory," Eur. Phys. J. C79 no. 3, (2019) 194. arXiv:1808.10169 [hep-th]

[140] Z.-Y. Fan and M. Guo, "Holographic complexity under a global quantum quench," arXiv:1811.01473 [hep-th]

[141] R. Abt, J. Erdmenger, M. Gerbershagen, C. M. Melby-Thompson, and C. Northe, "Holographic Subregion Complexity from Kinematic Space," JHEP 01 (2019) 012, arXiv:1805.10298 [hep-th].

[142] X. Huang and L. Zhang, "Holographic Complexity from the Crofton's Formula in Lorentzian $\mathrm{AdS}_{3}, "$ arXiv:1909.07048 [hep-th].

[143] Z.-Y. Fan and H.-Z. Liang, "The time dependence of complexity for Lovelock black holes," arXiv:1908.09310 [hep-th].

[144] R. J. Caginalp, "Holographic Complexity in FRW Spacetimes," arXiv:1906.02227 [hep-th]

[145] Y. Ling, Y. Liu, C. Niu, Y. Xiao, and C.-Y. Zhang, "Holographic Subregion Complexity in General Vaidya Geometry," arXiv:1908.06432 [hep-th].

[146] Y.-S. An, R.-G. Cai, L. Li, and Y. Peng, "Holographic complexity growth in a FRW universe," arXiv:1909.12172 [hep-th].

[147] J. Jiang and M. Zhang, "Holographic complexity of the electromagnetic black hole," arXiv:1905.07576 [hep-th].

[148] K. Goto, H. Marrochio, R. C. Myers, L. Queimada, and B. Yoshida, "Holographic Complexity Equals Which Action?," JHEP 02 (2019) 160 , arXiv:1901.00014 [hep-th].

[149] R. Auzzi, G. Nardelli, F. I. Schaposnik Massolo, G. Tallarita, and N. Zenoni, "On volume subregion complexity in Vaidya spacetime," JHEP 11 (2019) 098, arXiv:1908.10832 [hep-th]

[150] H.-S. Liu and H. Lu, "Action Growth of Dyonic Black Holes and Electromagnetic Duality," JHEP 09 (2019) 102, arXiv:1905.06409 [hep-th].

[151] P. Braccia, A. L. Cotrone, and E. Tonni, "Complexity in the presence of a boundary," arXiv:1910.03489 [hep-th].

[152] J. Jiang, B. Deng, and X.-W. Li, "Holographic complexity of charged Taub-NUT-AdS black holes," Phys. Rev. D100 no. 6, (2019) 066007, arXiv:1908.06565 [hep-th]

[153] H. E. Moumni and K. Masmar, "Testing the complexity conjecture in regular black holes geometry," Nuclear Physics B 950 (2020) 114837. http://www.sciencedirect.com/science/article/pii/S0550321319303232. 
[154] L. Vanzo, "Black holes with unusual topology," Phys. Rev. D56 (1997) 6475-6483, arXiv:gr-qc/9705004 [gr-qc].

[155] R. B. Mann, "Topological black holes: Outside looking in," Annals Israel Phys. Soc. 13 (1997) 311, arXiv:gr-qc/9709039 [gr-qc]. [,311(1997)].

[156] D. Birmingham, "Topological black holes in Anti-de Sitter space," Class. Quant. Grav. 16 (1999) 1197-1205, arXiv:hep-th/9808032 [hep-th].

[157] T. Torii and H. Maeda, "Spacetime structure of static solutions in Gauss-Bonnet gravity: Charged case," Phys. Rev. D72 (2005) 064007, arXiv:hep-th/0504141 [hep-th].

[158] S. H. Hendi, H. Behnamifard, and B. Bahrami-Asl, "Energy-dependent topological anti-de Sitter black holes in Gauss-Bonnet Born-Infeld gravity," Progress of Theoretical and Experimental Physics 2018 no. 3, $(03,2018)$, http://oup.prod.sis.lan/ptep/article-pdf/2018/3/033E03/24498041/pty017.pdf. https://doi.org/10.1093/ptep/pty017. 033E03.

[159] Y.-S. An, R.-G. Cai, and Y. Peng, "Time Dependence of Holographic Complexity in Gauss-Bonnet Gravity," Phys. Rev. D98 no. 10, (2018) 106013. arXiv:1805.07775 [hep-th]

[160] D. Klemm, V. Moretti, and L. Vanzo, "Rotating topological black holes," Phys. Rev. D57 (1998) 6127-6137, arXiv:gr-qc/9710123 [gr-qc]. [Erratum: Phys. Rev.D60,109902(1999)].

[161] S. Lloyd, "Ultimate physical limits to computation," Nature 406 (2000) 1047-1054, https://doi.org/10.1038/35023282.

[162] N. Margolus and L. B. Levitin, "The maximum speed of dynamical evolution," Physica D: Nonlinear Phenomena 120 (1998) 188-195. http://www.sciencedirect.com/science/article/pii/S0167278998000542.

[163] S. Lloyd, "Power of entanglement in quantum communication," Phys. Rev. Lett. 90 (Apr, 2003) 167902. https://link.aps.org/doi/10.1103/PhysRevLett.90.167902. 